University of Louisville

ThinkIR: The University of Louisville's Institutional Repository

Faculty Scholarship

$9-2015$

Reionization after Planck : the derived growth of the cosmic ionizing emissivity now matches the growth of the galaxy UV luminosity density.

\author{
R. J. Bouwens \\ Leiden University \\ G. D. Illingworth \\ University of California, Santa Cruz \\ P. A. Oesch \\ Yale University \\ J. Caruana \\ Leibniz-Institut fur Astrophysik Potsdam \\ Benne W. Holwerda \\ University of Louisville \\ See next page for additional authors
}

Follow this and additional works at: https://ir.library.louisville.edu/faculty

Part of the Astrophysics and Astronomy Commons

Original Publication Information

Bouwens, R. J., et al. "Reionization After Planck: The Derived Growth of the Cosmic lonizing Emissivity Now Matches the Growth of the Galaxy UV Luminosity Density." 2015. The Astrophysical Journal 811(2): $18 \mathrm{pp}$.

This Article is brought to you for free and open access by ThinkIR: The University of Louisville's Institutional Repository. It has been accepted for inclusion in Faculty Scholarship by an authorized administrator of ThinkIR: The University of Louisville's Institutional Repository. For more information, please contact thinkir@louisville.edu. 


\section{Authors}

R. J. Bouwens, G. D. Illingworth, P. A. Oesch, J. Caruana, Benne W. Holwerda, R. Smit, and S. M. Wilkins 


\title{
REIONIZATION AFTER PLANCK: THE DERIVED GROWTH OF THE COSMIC IONIZING EMISSIVITY NOW MATCHES THE GROWTH OF THE GALAXY UV LUMINOSITY DENSITY*
}

\author{
R. J. Bouwens ${ }^{1,2}$, G. D. Illingworth ${ }^{2}$, P. A. Oesch ${ }^{3}$, J. Caruana ${ }^{4}$, B. Holwerda ${ }^{1}$, R. Smit $^{5}$, and S. Wilkins ${ }^{6}$ \\ ${ }^{1}$ Leiden Observatory, Leiden University, NL-2300 RA Leiden, The Netherlands \\ ${ }^{2}$ UCO/Lick Observatory, University Of California, Santa Cruz, CA 95064, USA \\ ${ }^{3}$ Department of Astronomy, Yale University, New Haven, CT 06520, USA \\ ${ }^{4}$ Leibniz-Institut fur Astrophysik Potsdam (AIP), An der Sternwarte 16, D-14482 Potsdam, Germany \\ ${ }^{5}$ Department of Physics and Astronomy, South Road, Durham, DH1 3EE, UK \\ ${ }^{6}$ Department of Physics \& Astronomy, University of Sussex, Falmer, Brighton, BN1 9QH, UK \\ Received 2015 March 29; accepted 2015 August 9; published 2015 September 30
}

\begin{abstract}
Thomson optical depth $\tau$ measurements from Planck provide new insights into the reionization of the universe. In pursuit of model-independent constraints on the properties of the ionizing sources, we determine the empirical evolution of the cosmic ionizing emissivity. We use a simple two-parameter model to map out the evolution in the emissivity at $z \gtrsim 6$ from the new Planck optical depth $\tau$ measurements, from the constraints provided by quasar absorption spectra and from the prevalence of $\operatorname{Ly} \alpha$ emission in $z \sim 7-8$ galaxies. We find the redshift evolution in the emissivity $\dot{N}_{\text {ion }}(z)$ required by the observations to be $\left(d \log _{10} \dot{N}_{\text {ion }} / d z\right)_{z=8}=-0.15_{-0.11}^{+0.08}$ $\left(\left(d \log _{10} \dot{N}_{\text {ion }} / d z\right)_{z=8}=-0.19_{-0.11}^{+0.09}\right.$ for a flat prior), largely independent of the assumed clumping factor $C_{\mathrm{H} \text { II }}$ and entirely independent of the nature of the ionizing sources. The trend in $\dot{N}_{\text {ion }}(z)$ is well-matched by the evolution of the galaxy UV-luminosity density $\left(d \log _{10} \rho_{\mathrm{UV}} / d z=-0.11 \pm 0.04\right)$ to a magnitude limit $\gtrsim-13 \mathrm{mag}$, suggesting that galaxies are the sources that drive the reionization of the universe. The role of galaxies is further strengthened by the conversion from the UV luminosity density $\rho_{\mathrm{UV}}$ to $\dot{N}_{\text {ion }}(z)$ being possible for physically plausible values of the escape fraction $f_{\text {esc }}$, the Lyman-continuum photon production efficiency $\xi_{\text {ion }}$, and faint-end cut-off $M_{\text {lim }}$ to the luminosity function. Quasars/active galactic nuclei appear to match neither the redshift evolution nor normalization of the ionizing emissivity. Based on the inferred evolution in the ionizing emissivity, we estimate that the $z \sim 10$ UV-Iuminosity density is $8_{-4}^{+15} \times$ lower than at $z \sim 6$, consistent with the observations. The present approach of contrasting the inferred evolution of the ionizing emissivity with that of the galaxy UV luminosity density adds to the growing observational evidence that faint, star-forming galaxies drive the reionization of the universe.
\end{abstract}

Key words: galaxies: evolution - galaxies: high-redshift

\section{INTRODUCTION}

One of the most important phase transitions in the history of the universe is the reionization of the neutral hydrogen gas. Following recombination early in the universe shortly after the Big Bang, the universe likely remained in a largely neutral state until $z \sim 15-25$. The collapse of the first dark matter halos and gas cooling brought about the formation of the first stars and galaxies. These early stars and galaxies have long been thought to provide the ionizing photons necessary to reionize the universe (Loeb \& Barkana 2001; Loeb 2006).

Despite this general picture of reionization and the likely role that early galaxies played in the process, establishing that this is the case has been particularly challenging, both due to the difficulties in probing the ionization state of the universe at $z>6$ (e.g., Ouchi et al. 2010; Stark et al. 2010) and providing constraints on the ionizing photons that early galaxies themselves are thought to produce (e.g., Siana et al. 2010, 2015; Vanzella et al. 2012; Mostardi et al. 2013; Nestor et al. 2013; Cooke et al. 2014). Further complicating the interpretation were early measurements of the Thomson optical depth by $W M A P$ (e.g., $\tau=0.17 \pm 0.06$ : Spergel et al. 2003) which suggested a significant quantity of the ionizing photons in the

\footnotetext{
* Based on observations made with the NASA/ESA Hubble Space Telescope, which is operated by the Association of Universities for Research in Astronomy, Inc., under NASA contract NAS 5-26555.
}

early universe-implying an instantaneous reionization redshift of $z_{r}=20_{-9}^{+11}$ (95\% confidence) - and which showed no clear connection with the early evolution of galaxies. While lower optical depths were measured in subsequent studies by WMAP (i.e., $\tau=0.089 \pm 0.014$ : Bennett et al. 2013) with an implied $z_{r}=10.6 \pm 1.1$, these depths still pointed toward substantial amounts of ionizing radiation being present in the early universe.

Fortunately, substantial progress has been made over the last 10 years to better understand cosmic reionization. Much of the progress has been observational, through the better study of bright quasars and improved statistics on $\operatorname{Ly} \alpha$ emission in normal star-forming galaxies, to better probe the ionization state of the $z=6-9$ universe (e.g., Mortlock et al. 2011; Ono et al. 2012; Pentericci et al. 2014 [P14]; Caruana et al. 2014; Schenker et al. 2014 [S14]). The greater depths of probes for $z=6-10$ galaxies provided greater confidence that galaxies could provide the necessary reservoir of photons to reionize the universe (e.g., Bouwens et al. 2010, 2011, 2015 [B15]; Bunker et al. 2010; McLure et al. 2013; Oesch et al. 2010, 2013; Ellis et al. 2013). However, theoretical progress has been similarly substantial, due to significantly improved estimates of the clumping factor (Bolton \& Haehnelt 2007; Pawlik et al. 2009, 2015; Finlator et al. 2012; Shull et al. 2012) and ever more sophisticated simulations tracking the reionization of the universe and the propagation of $\operatorname{Ly} \alpha$ out of the galaxies and into the intergalactic medium (IGM) (e.g., Choudhury et al. 
2015; Mesinger et al. 2015). Lastly, measurements of the integrated column of ionized material to the last-scattering surface from the three-year Planck mission yield $\tau=0.066 \pm$ 0.013 (Planck Collaboration et al. 2015, XIII [PC15]), implying that $z_{r}=8.8_{-1.2}^{+1.3}$ and suggesting that current surveys may be uncovering the sources that led to the reionization of the universe.

Naturally, numerous studies have taken advantage of this collective progress to construct self-consistent models for reionizing the universe (e.g., Choudhury \& Ferrara 2006; Bolton \& Haehnelt 2007; Oesch et al. 2009; Trenti et al. 2010; Alvarez et al. 2012; Bouwens et al. 2012a; Finkelstein et al. 2012a; Haardt \& Madau 2012 [HM12]; Kuhlen \& FaucherGiguère 2012 [KF12]; Shull et al. 2012; Robertson et al. 2013 [R13], 2015 [R15]; Cai et al. 2014; Choudhury et al. 2015; Ishigaki et al. 2015), and it is indeed encouraging that many recent models (e.g., R15) prove successful in reionizing the universe, while matching many other individual constraints on the reionization state of the universe and also the consensus star formation history (e.g., Madau \& Dickinson 2014; B15).

While these analyses are reassuring and offer strong support for the idea that galaxies reionize the universe, the uniqueness of galaxies as the source of photons to reionize the universe is more challenging to establish. Indeed, it is possible to imagine the existence of other populations of ionizing sources (e.g., Chen et al. 2003; Somerville et al. 2003; Hansen \& Haiman 2004; Madau et al. 2004; Ricotti \& Ostriker 2004; Ricotti et al. 2008), which even if speculative could also match current constraints.

What model-independent statements can be made about the sources that reionize the universe? To answer this question, it is useful to look at the evolution of the cosmic ionizing emissivity, since this allows us to keep assumptions regarding the nature of the ionizers to a minimum. As we will show, interesting constraints on the evolution of the emissivity can be obtained based on current observations, e.g., from the Thomson optical depths or the inferred filling factor of ionized hydrogen $Q_{\mathrm{H} \text { II }}$ at $z=6-9$ (see also Mitra et al. 2011, 2012, 2013).

The purpose of the present analysis is to take advantage of current observational constraints on the ionization state of the universe at $z>6$ (e.g., Fan et al. 2006b; S14; PC15) to constrain the evolution of the cosmic ionizing emissivity with redshift. Through comparisons with the evolution of the UV luminosity density of galaxies and other potential ionizing sources, we can evaluate the likelihood that each of these sources of ionizing photons drives the reionization of the universe. We begin with a description of the relevant observations (Section 2) and methodology (Section 3) and then derive constraints on the evolution of the ionizing emissivity $\dot{N}_{\text {ion }}$ (Section 4.1). After deriving constraints on $\dot{N}_{\text {ion }}(z)$, we compare our results with what we would expect for galaxies (Section 4.2), quasars (Section 4.3), and consider the implications for the UV luminosity density at $z \sim 10$ (Section 4.4). We conclude with a brief summary (Section 5). We take $H_{0}=67.51 \pm 0.64, \Omega_{\Lambda}=0.6879 \pm 0.0087$, and $\Omega_{m}=0.3121 \pm 0.0087, \Omega_{b} h^{2}=0.02230 \pm 0.00014(\mathrm{PC} 15)$.

\section{OBSERVATIONAL CONSTRAINTS}

There are a wide variety of observational constraints in the literature on the ionization state of neutral hydrogen that can be leveraged in considering questions regarding the reionization of the universe.
In the present analysis, we consider constraints at four different points in the reionization history of the universe. First of all, there are useful constraints on the end of cosmic reionization at $z=5.0-6.5$. The most important constraints make use of the Gunn-Peterson optical depth measured from bright $z \sim 6$ quasars (Becker et al. 2001; Fan et al. 2006a, $2006 \mathrm{~b}$ ) and also by looking at the distribution of dark gaps in $z=6$ quasar spectra (McGreer et al. 2015) which suggest that cosmic reionization is complete by $z=5.9$. By contrast, the presence of damping absorption wings in the spectra of three $z=6.2-6.4$ quasars studied by Schroeder et al. (2013) strongly suggest that cosmic reionization is not complete by $z=6.4$. These results clearly indicate that cosmic reionization is completed between $z=6.5$ and $z=5.9$.

Second, we consider the constraints on the reionization history of the universe that come from the prevalence of Ly $\alpha$ emission in the UV spectra of $z \sim 6-8$ galaxies. Assuming that the prevalence of Ly $\alpha$ emission in star-forming galaxies at $z \sim$ $6.5-8$ is a simple extrapolation of the observed prevalence at $z$ $\sim 4-6$ and any departures from these trends are due to an increasingly neutral IGM at $z \sim 7-8$, one can use this technique to quantify the filling factor of ionized hydrogen at $z \sim 6.5-8$ (Malhotra \& Rhoads 2004; Santos 2004; McQuinn et al. 2007; Mesinger \& Furlanetto 2008; Fontana et al. 2010; Stark et al. 2010, 2011; Dijkstra et al. 2011; Pentericci et al. 2011; Caruana et al. 2012, 2014; Ono et al. 2012; Treu et al. 2013; Tilvi et al. 2014; but see also Bolton \& Haehnelt 2013; Mesinger et al. $2015)$. Here, we will make use of the recent constraints on $Q_{\mathrm{H} \text { II }}$ from $\mathrm{S} 14$, i.e., $Q_{\mathrm{H} \text { II }}(z=7)=0.66_{-0.12}^{+0.09}$ and $Q_{\mathrm{H} \text { II }}(z=8)<$ 0.35, using the McQuinn et al. (2007) models, but also briefly consider the impact of an alternate set of constraints.

Of course, the real uncertainties on $Q_{\mathrm{H} \text { II }}$ are likely larger than the formal uncertainties quoted by $\mathrm{S} 14$, as can be seen by comparing the different estimates for $Q_{\mathrm{H} \text { II }}$ quoted by $\mathrm{S} 14$ based on the same set of observations or using the results from other studies (Pentericci et al. 2014; Tilvi et al. 2014). Therefore, we take the formal $1 \sigma$ uncertainty in $Q_{\mathrm{H} \text { II }}$ to be \pm 0.15 , such that $Q_{\mathrm{H} \text { II }}(z=7)=0.66 \pm 0.15$. Consistent with the constraints from $\mathrm{S} 14$, we suppose that $Q_{\mathrm{H} \text { II }}(z=8)$ is equal to $0.15 \pm 0.20$. However, we recognize that the constraints we use are just estimates and the true values could be different if the assumptions used in deriving these fractions are not correct (e.g., regarding the velocity offset for Ly $\alpha$ line or the photoionization rate $\Gamma(z)$ : Mesinger et al. 2015).

Third, we consider the constraints on the cosmic ionizing emissivity that come from the Thomson optical depth $\tau=0.066 \pm 0.013$ measured from the three-year Planck results (PC15). While this optical depth measurement does not provide any information on the filling factor of ionized hydrogen at a specific time, it does provide a powerful constraint on the integrated path length of ionized hydrogen to the last-scattering surface.

Finally, we require that the ionizing emissivity extrapolated to $z=4.75$ be consistent with the $10^{50.99 \pm 0.45} \mathrm{~s}^{-1} \mathrm{Mpc}^{-3}$ measurement derived by Becker \& Bolton (2013: BB13) based on observations of the $\operatorname{Ly} \alpha$ forest by considering a wide variety of systematics on this measurement. Observations of the Ly $\alpha$ forest allow for constraints on the emissivity through the impact of the photoionization rate $\Gamma(z)$ on the prevalence and statistics of Ly $\alpha$-forest clouds (and also from constraints on the mean-free path $\lambda_{\mathrm{mfp}}$ using similar observations). 
Table 1

Key Observational Constraints on the Reionization History of the Universe ${ }^{\mathrm{a}}$

\begin{tabular}{|c|c|c|c|c|}
\hline $\begin{array}{l}\text { Constraint } \\
\#\end{array}$ & Redshift & $Q_{\mathrm{H} \mathrm{II}}$ Constraint & Technique & References \\
\hline \multicolumn{5}{|c|}{$\begin{array}{l}\text { Key Constraints on the Ionization History of the Universe Explicitly Considered Here } \\
\text { In Deriving the Evolution of the Cosmic Ionizing Emissivity at } z>6\end{array}$} \\
\hline 1. & \multicolumn{3}{|c|}{ Thomson Optical Depth $\tau=0.066 \pm 0.013$} & PC15 \\
\hline 2. & \multicolumn{4}{|c|}{ Reionization Finishes Between $z=5.9$ and $z=6.5$} \\
\hline & 5.03 & $0.999945+0.00000165$ & Gunn-Peterson Optical Depth & Fan et al. (2006b) \\
\hline & 5.25 & $0.999933+0.0000207$ & Gunn-Peterson Optical Depth & Fan et al. (2006b) \\
\hline & 5.45 & $0.999934+0.00000247$ & Gunn-Peterson Optical Depth & Fan et al. (2006b) \\
\hline & 5.65 & $0.999912+0.0000365$ & Gunn-Peterson Optical Depth & Fan et al. (2006b) \\
\hline & 5.85 & $0.99987+0.0000408$ & Gunn-Peterson Optical Depth & Fan et al. (2006b) \\
\hline & 6.10 & $0.99957 \pm 0.00030$ & Gunn-Peterson Optical Depth & Fan et al. (2006b) \\
\hline & 5.9 & $>0.89$ & Dark Gaps in Quasar Spectra & McGreer et al. (2015) \\
\hline & 5.6 & $>0.91$ & Dark Gaps in Quasar Spectra & McGreer et al. (2015) \\
\hline & $6.24-6.42$ & $<0.9(2 \sigma)$ & Ly $\alpha$ Damping Wing of Quasars & Schroeder et al. (2013) \\
\hline \multicolumn{5}{|c|}{ Higher-Redshift Constraints } \\
\hline 3. & 7.0 & $Q_{\mathrm{H} \mathrm{II}}(z=7)=0.66_{-0.09}^{+0.12}$ & Prevalence of Ly $\alpha$ Emission in Galaxies & S14 \\
\hline 4. & 8.0 & $Q_{\mathrm{H} \mathrm{II}}(z=8)<0.35$ & Prevalence of Ly $\alpha$ Emission in Galaxies & S14 \\
\hline 5. & & $\begin{array}{l}\text { Continuity with Ion } \\
\log _{10} \dot{N}_{\text {ion }}(z=4.75)\end{array}$ & $\begin{array}{l}\text { Ivity Estimates at } z=4.75 \\
\mathrm{~s}^{-1} \mathrm{Mpc}^{-3}\end{array}$ & BB13 \\
\hline \multicolumn{5}{|c|}{ Other Constraints on the Ionization History of the Universe Not Explicitly Used ${ }^{\mathrm{b}}$} \\
\hline & 6.3 & $\geqslant 0.5$ & Ly $\alpha$ Damping Wing of a GRB & $\begin{array}{l}\text { Totani et al. (2006) } \\
\text { McQuinn et al. (2008) }\end{array}$ \\
\hline & 6.6 & $\geqslant 0.6$ & Ly $\alpha$ Emitters & Ouchi et al. (2010) \\
\hline & 6.6 & $\geqslant 0.5$ & Galaxy Clustering & $\begin{array}{l}\text { McQuinn et al. (2007), } \\
\text { Ouchi et al. (2010) }\end{array}$ \\
\hline & 7.0 & $0.32-0.64$ & Ly $\alpha$-Emitter LFs & Ota et al. (2008) \\
\hline & 7.0 & $\sim 0.5$ & Prevalence of $\operatorname{Ly} \alpha$ Emission in Galaxies & Caruana et al. (2014) \\
\hline & 7.0 & $0.1-0.4$ & Prevalence of Ly $\alpha$ Emission in Galaxies & Ono et al. (2012) \\
\hline & 7.0 & $<0.49$ & Prevalence of Ly $\alpha$ Emission in Galaxies & P14 \\
\hline & 7.0 & $<0.5$ & Prevalence of Ly $\alpha$ Emission in Galaxies & $\mathrm{R} 13^{\mathrm{c}}$ \\
\hline & 7.0 & $<0.5$ & Clustering of Ly $\alpha$ Emitting Galaxies & $\begin{array}{c}\text { Sobacchi \& } \\
\text { Mesinger }(2015)\end{array}$ \\
\hline & 7.1 & $\leqslant 0.9$ & Near-zone Quasar & $\begin{array}{l}\text { Mortlock et al. (2011), } \\
\text { Bolton et al. (2011) }\end{array}$ \\
\hline & 8.0 & $<0.70$ & Prevalence of Ly $\alpha$ Emission in Galaxies & Tilvi et al. (2014) \\
\hline
\end{tabular}

Notes.

${ }^{\mathrm{a}}$ This table is a compilation of the constraints presented in the original papers under References, but with valuable guidance by the results presented in Figures 5 and 3 from R13 and R15, respectively.

${ }^{\mathrm{b}}$ While not explicitly considered in deriving the evolution of the cosmic ionizing emissivity, almost all of these constraints are satisfied for the typical reionization histories derived in this study (see right panel of Figure 9).

${ }^{\mathrm{c}} \mathrm{R} 13$ estimate this constraint on $Q_{\mathrm{H} \text { II }}$ based on the observational results from Fontana et al. (2010), Pentericci et al. (2011), Schenker et al. (2012), and Ono et al. (2012) and the simulation results from McQuinn et al. (2007), Mesinger \& Furlanetto (2008), and Dijkstra et al. (2011).

We summarize all four of these key constraints in Table 1 and Figure 1, along with other constraints that have been derived in the literature. We refer interested readers to R13 and R15 for a comprehensive summary of these constraints.

\section{MODELING THE EVOLUTION OF THE FILLING FACTOR OF IONIZED HYDROGEN $Q_{\mathrm{H} \mathrm{II}}$}

Here we describe the simple evolutionary models we consider for the evolution of the cosmic ionizing emissivity and which we will compare against observational constraints on the ionization state of the universe.

We model the evolution of the cosmic ionizing emissivity $\dot{N}_{\text {ion }}(z)$ using just two parameters $A$ and $B$ :

$$
\dot{N}_{\text {ion }}(z)=A 10^{B(z-8)}
$$

where $A=\dot{N}_{\text {ion }}(z=8)$ and $B=\left(d \log _{10} \dot{N}_{\text {ion }} / d z\right)_{z=8}$. As in previous analyses (e.g., Madau et al. 1999; Bolton \&
Haehnelt 2007; KF12), we follow the evolution of $Q_{\mathrm{H} \text { I }}$ using the relation

$$
\frac{d Q_{\mathrm{H} \mathrm{II}}}{d t}=\dot{N}_{\text {ion }}(z)-\frac{Q_{\mathrm{H} \mathrm{II}}}{t_{\text {rec }}}
$$

until $Q_{\mathrm{H} \mathrm{II}}=1$ when this equation ceases to be valid and the ionizing emissivity impacts not only atomic-hydrogen gas in the IGM, but also that in higher-density Ly $\alpha$ clouds. We take $Q_{\mathrm{H} \mathrm{II}}=0$ at $z=25$. The recombination time $t_{\text {rec }}$ is as follows (KF12):

$$
t_{\mathrm{rec}}=0.88 \mathrm{Gyr}\left(\frac{1+z}{7}\right)^{-3}\left(\frac{T_{0}}{2 \times 10^{4} \mathrm{~K}}\right)^{-0.7}\left(C_{\mathrm{H} \mathrm{II}} / 3\right)^{-1}
$$

where $C_{\mathrm{H} \text { II }}$ is the clumping factor of ionized hydrogen $\left\langle n_{\mathrm{H} \mathrm{III}}^{2}\right\rangle /\left\langle n_{\mathrm{H} \mathrm{III}}\right\rangle^{2}$ and $T_{0}$ is the temperature of the ionizing hydrogen gas. We adopt a value of $2 \times 10^{4} \mathrm{~K}$ for the 


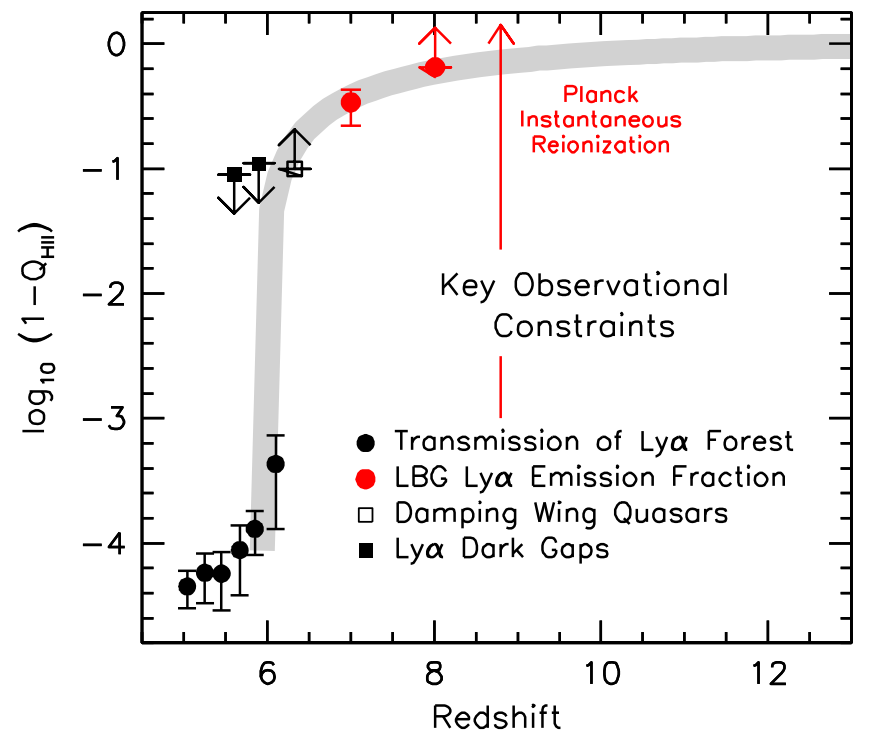

Figure 1. The key observational constraints on the filling factor of ionized hydrogen $Q_{\mathrm{H} \text { II }}$ considered here in modeling the evolution of the cosmic ionizing emissivity (Section 2 and Table 1). These constraints include the Gunn-Peterson optical depths and dark-gap statistics measured in $z \sim 6$ quasars (Fan et al. 2006b; McGreer et al. 2015), damping wings measured in $z$ 6.2-6.4 quasars (Schroeder et al. 2013), and the prevalence of Ly $\alpha$ emission in $z \sim 7-8$ galaxies (S14). Based on the constraints shown in this figure (particularly on the basis of the Gunn-Peterson troughs, dark-gap statistics, and damping wings of $z \sim 6-6.5$ quasars), reionization ends between $z=5.9$ and $z=6.5$. The other key constraints we consider are the new Thomson optical depth measurements from Planck (PC15: represented here with an upwardpointing red arrow at the instantaneous reionization redshift $z_{\text {reion }}=8.8_{-1.2}^{+1.3}$ ) and a requirement for continuity with the cosmic ionizing emissivity at $z=4.75$, as derived by BB13. The large changes in the filling factor are represented schematically by the gray-shaded region.

temperature $T_{0}$ of the ionizing gas to account for the heating of the gas that occurs due to the reionization process itself (Hui \& Haiman 2003).

Our calculation of the Thomson optical depths $\tau$ themselves also follow familiar expressions from previous analyses (e.g., KF12):

$$
\tau=\int_{0}^{\infty} d z \frac{c(1+z)^{2}}{\mathrm{H}(z)} Q_{\mathrm{H} \text { II }}(z) \sigma_{T} \bar{n}_{\mathrm{H}}(1+\eta Y / 4 X)
$$

where $\sigma_{\mathrm{T}}$ is Thomson cross section and $X$ and $Y$ is the primordial mass fraction of hydrogen and helium. Following KF12, we assume that helium is singly ionized at $z>4(\eta=1)$ and doubly ionized at $z<4(\eta=2)$.

\section{RESULTS}

\subsection{Quantifying the Redshift Evolution of the Cosmic Ionizing Emissivity}

Here we examine what constraints can be set on the evolution of the cosmic ionizing emissivity from the key observational constraints we consider. We can do this without knowledge of the nature of the ionizing sources, due to the simplicity of the basic equations that govern cosmic reionization, i.e., Equations (2) and (3). Nothing in these equations requires knowledge of the nature of the ionizing sources.

To derive constraints on the ionizing emissivity, we consider a full two-dimensional grid of plausible values of (1) the normalization of the ionizing emissivity, i.e., $\log _{10} \dot{N}_{\text {ion }}(z)_{z}=8$ (from 49.7 to $51.5 \mathrm{~s}^{-1} \mathrm{Mpc}^{-3}$ ) and (2) the dependence of this emissivity on redshift, i.e., $\left(d \log _{10} \dot{N}_{\text {ion }} / d z\right)_{z=8}$ (i.e., from -0.6 to 0.1 ). For each choice of the normalization and redshift dependence of the ionizing emissivity ( $A$ and $B$ in Equation (1)), we investigate whether the assumed emissivity would produce a reionization history and Thomson optical depth (computed from a reionization history using Equation (4)) consistent with the key observables we consider (i.e., constraints $1-5$ in Table 1 and Figure 1).

We begin by considering the model ionizing emissivities allowed assuming a redshift-independent clumping factor of 3 , but then later explore what the impact would be of different clumping factors, as well as considering clumping factors that evolve with redshift. Our choice of 3 for the fiducial value of the clumping factor is motivated by the results of Bolton \& Haehnelt (2007) and Pawlik et al. (2009: see also Finlator et al. 2012 and Shull et al. 2012).

To illustrate the impact that various observational constraints have on the evolution of the cosmic ionizing emissivities, we start by considering only a subset of the available constraints. More specifically, we consider the impact of matching both the Thomson optical depths of different microwave background missions (best estimate and $\pm 1 \sigma$ ) and requiring that reionization be complete between $z=5.9$ and $z=6.5$ (i.e., when $Q_{\mathrm{H} \text { II }}$ first reaches 1 using Equation (2)), while taking $C_{\mathrm{H}_{\text {II }}}=3$. Section 2 describes the rationale for the $z_{\text {reion }}=5.9-6.5$ constraint. The results are shown in the top two panels of Figure 2.

As the top two panels illustrate, there are clear differences between the implied evolution of ionizing sources for WMAP and Planck. Similar to previous models of the ionizing emissivity which approximately match the WMAP constraints (e.g., Haardt \& Madau 2012; KF12), the ionizing emissivity we derive shows no (or a slightly declining) evolution from $z \sim 11$. For the Planck constraints on $\tau$, a range of different models are possible, though the ionizing emissivity in all acceptable models increases with cosmic time.

Interestingly enough, the allowed evolution of the cosmic ionizing emissivity with redshift shows excellent continuity with the emissivity inferred at $z=3-5$ from the Ly $\alpha$ forest by Becker \& Bolton (2013) [BB13]. This suggests that the evolution we have derived for the ionizing emissivity is plausible. $^{7}$

In Figure 2, we only present our constraints on the ionizing emissivity at redshifts $z=6-12$ where changes in the filling factor of ionized hydrogen will affect the observations we consider. At redshifts lower than $z \sim 6$, the IGM is almost entirely ionized, and any change in the ionizing emissivity will have little impact on the $Q_{\mathrm{H} \text { II }}(z)$ 's we consider or the Thomson optical depths.

More detailed constraints on the evolution can be achieved by examining estimates of the filling factor of ionizing hydrogen $Q_{\mathrm{H} \text { II }}$ derived from studies of the prevalence of Ly $\alpha$ emission in $z \sim 7$ and $z \sim 8$ galaxies and by requiring that the

\footnotetext{
7 While the emissivity we derive (extrapolated to $z \sim 4$ ) agrees with BB13, it agrees less well with that inferred by KF12 (indicated by the hatched-dotted gray region in the top left panel of Figure 2). This is due to a tension between the ionizing emissivity results of KF12 and BB13. In noting this tension, readers should realize that all such determinations of this emissivity depend quite sensitively on various model parameters like the temperature of the IGM and opacity to ionizing photons. For BB13's determination of the ionizing emissivity, it was possible to take advantage of some new measurements of the IGM temperature over the redshift range $z=2.0-4.8$ (Becker et al. 2011), while also including an account for cosmological radiative transfer effects.
} 


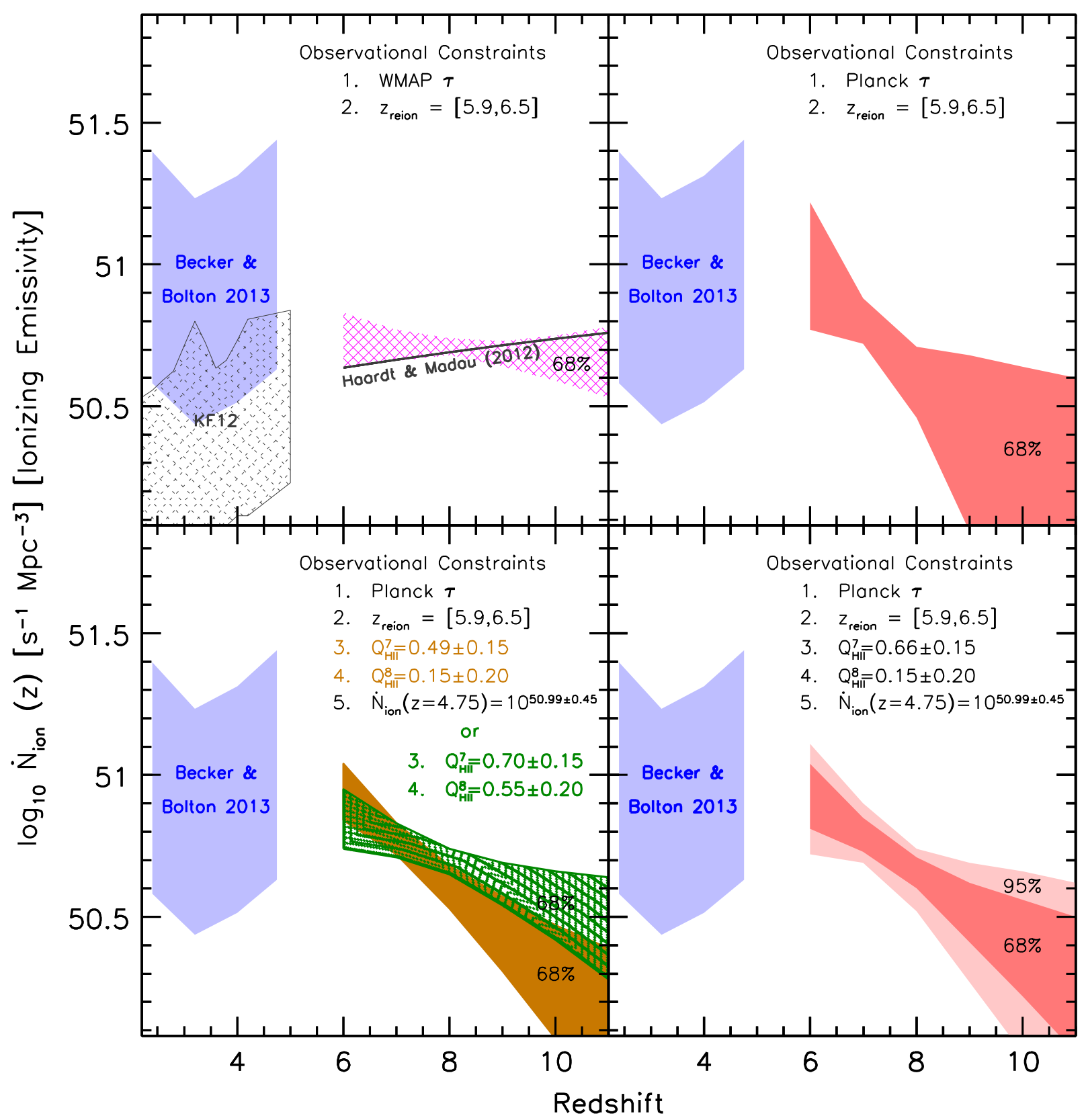

Figure 2. Allowed evolution ( $68 \%$ confidence) of the cosmic ionizing emissivity over the redshift range $z=6$ to $z=11$, as inferred from the observations. (Upper left) Constraints on the evolution of the emissivity supposing a completion of reionization at $z=5.9-6.5$ (Fan et al. 2006b; Schroeder et al. 2013; McGreer et al. 2015) and the nine-year measured Thomson optical depth $\tau=0.089 \pm 0.14$ from WMAP (Bennett et al. 2013), and a simple modeling of the evolution of $Q_{\mathrm{H} \text { п }}$ with $C_{\mathrm{H} \text { п }}=3$. The region so defined is presented in hatched magenta. Constraints on the emissivity from $z=2$ to $z=5$ from BB13 are shown with the light-blue-shaded region and were derived from observations of the Ly $\alpha$ forest. Systematic uncertainties in the inferred emissivity can often be large, $\sim 0.3$ dex, due to the sensitivity to the assumed (or measured) temperature of the IGM and the opacity to ionizing photons. As a result, some past constraints have been much lower than those from BB13 (e.g., KF12: dotted-gray-hatched region). The light gray line shows the ionizing emissivity model from HM12 constructed to match the WMAP $\tau$ 's (and the models from KF12 are similar to this [see the lower panel of Figure 10 from KF12]). (Upper right) Identical to the upper-left panel except including the optical depth constraints from Planck (PC15: red-shaded region). (Lower left) Constraints on the evolution of the ionizing emissivity (orange-shaded and green-shaded regions) assuming a completion of reionization at $z=5.9-6.5$, the Planck Thomson optical depths, and various constraints on $Q_{\mathrm{H} \text { ㅍ }}$ using the prevalence of Ly $\alpha$ emission in candidate $z \sim 7-8$ galaxies. (Lower right) Similar to the lower left panel, but using the $Q_{\mathrm{H} \text { ш }}$ constraints from S14 (red-shaded region) and also showing the $95 \%$ confidence intervals. The 68\% and 95\% confidence intervals featured in this panel are also expressed in tabular form in Table 2.

evolution of the ionizing emissivity be consistent (at $1 \sigma$ ) with the $z=4.75$ constraints from BB13. In the lower two panels of Figure 2, the allowed evolution $(68 \%$ [left+right] and $95 \%$ [right] confidence) in $\dot{N}_{\text {ion }}(z)$ is shown based on $Q_{\mathrm{H} \text { п }}$ 's derived in S14 (as specified in Section 2) and also allowing for a higher value of $Q_{\mathrm{H} \text { II }}=0.49 \pm 0.15$ at $z \sim 7$ (e.g., as in Caruana et al. 2014) and $Q_{\mathrm{H} \mathrm{II}}=0.55 \pm 0.20$ at $z \sim 8$. The confidence regions here are derived by calculating the probabilities based on constraints 1 and 3-5 (Table 1) and then marginalizing over the full two-dimensional $\log _{10} \dot{N}_{\text {ion }}(z=8)$ versus $\left(d \log _{10} \dot{N}_{\text {ion }} / d z\right)_{z=8}$ space based on the total $\chi^{2}$ computed from constraints 1 and 3-5 (Table 1). In marginalizing over the parameter space, regions where $z_{\text {reion }}<5.9, z_{\text {reion }}>6.5$, and $\log _{10} \dot{N}_{\text {ion }}(z=4.75)>51.44$ are excluded. Individual regions 


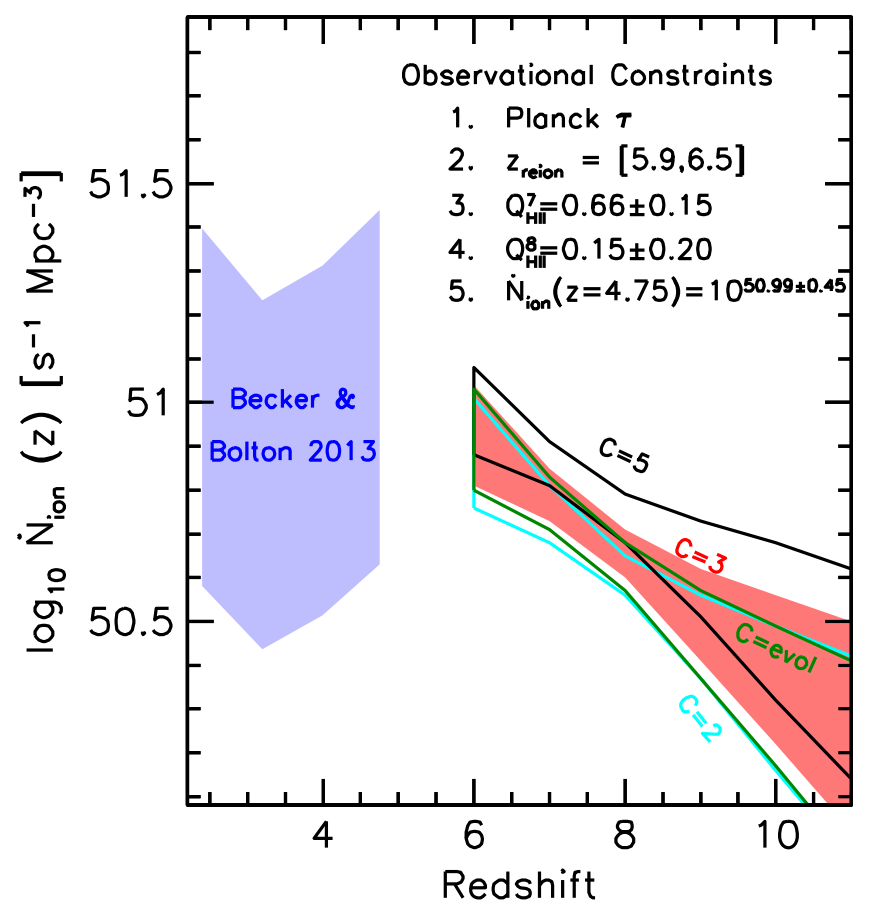

Figure 3. Allowed evolution (68\% confidence) of the cosmic ionizing emissivity (region demarcated by the cyan lines, red-shaded region, and regions demarcated by the black and green lines) over the redshift range $z=6$ to $z=11$, as inferred from the three primary observational constraints considered here (Fan et al. 2006b; S14; PC15) and assuming the clumping factor $C_{\mathrm{H}}$ ІІ remains fixed at $2,3,5$, and evolves as $1+43 z^{-1.71}$ (as found by the hydrodynamical simulations of Pawlik et al. 2009), respectively. The lightblue-shaded region indicate the constraints from BB13. The normalization of the derived ionizing emissivity only shows a weak dependence on the assumed value of the clumping factor $C_{\mathrm{H}_{\text {II }}}$, changing by just $\sim 30 \%$ for factor-of- 2.5 differences in $C_{\mathrm{H}_{\text {II }}}$ (i.e., $C_{\mathrm{H} \text { II }}=2$ vs. $C_{\mathrm{H}_{\text {II }}}=5$ ). Strikingly, the evolution inferred for the ionizing emissivity, i.e., $\left(d \log _{10} \dot{N}_{\text {ion }} / d z\right)_{z=8}$, is even less sensitive to the adopted value for the clumping factor (see also Table 3).

of parameter space are weighted according to the calculated $|\nabla \tau|$ (where the derivatives are with respect to $\log _{10} \dot{N}_{\text {ion }}$ and $\left.\left(d \log _{10} \dot{N}_{\text {ion }} / d z\right)_{z=8}\right)$. In doing so, we weight regions of parameter space according to the impact they have on physical variables like the Thomson optical depth $\tau$ (which approximately varies according to the total output of ionizing photons at $z \sim 8-15)$ and give less weight to those regions in parameter space (i.e., $\left.\left(d \log _{10} \dot{N}_{\text {ion }} / d z\right)_{z=8}<-0.3\right)$ which have less impact on the ionizing emissivity at $z>>6$. The $68 \%$ and $95 \%$ confidence intervals presented in the lower right panel of Figure 2 are tabulated in Table 2.

It is clear that if the universe is only $50 \%$ ionized by $z \sim 8$ (in significant contrast to the results of S14), it would imply a much higher cosmic ionizing emissivity at $z \geqslant 8$. If we assume that the universe is measured to be even more ionized at $z \sim 7$ and $z \sim 8$, i.e., $Q_{\mathrm{H}_{\text {II }}}=0.9$, as an even more extreme example (this situation might arise if use of the $\operatorname{Ly} \alpha$ fraction as a probe of $Q_{\mathrm{H} \text { II }}$ is subject to large systematic errors: e.g., Mesinger et al. 2015: but note also Keating et al. 2014 and Sobacchi \& Mesinger 2015), we derive $\log _{10} \dot{N}_{\text {ion }}(z=8)=50.74_{-0.03}^{+0.02}$ and $\left(d \log _{10} \dot{N}_{\text {ion }} / d z\right)_{z=8}=-0.03_{-0.04}^{+0.03}$. The inferred cosmic ionizing emissivity in this case is 0.07 dex higher at $z \sim 8$ and 0.31 dex higher at $z \sim 10$ than our fiducial determination.

We found it challenging to reproduce the $<0.3$ filling factors of ionized hydrogen $Q_{\mathrm{H}}$ i at $z \sim 7$ found by Ono et al. (2012) within the context of our simple model for the ionizing
Table 2

$68 \%$ and $95 \%$ Confidence Intervals on the Inferred Ionizing Emissivity vs. Redshift for a Fiducial Clumping factor $C_{\mathrm{H} \text { II }}$ of 3

\begin{tabular}{lcccr}
\hline \hline & \multicolumn{4}{c}{$\begin{array}{c}\log _{10} \dot{N}_{\text {ion }}\left(\mathrm{s}^{-1} \mathrm{Mpc}^{-3}\right) \\
\text { Lower Bound }\end{array}$} \\
Redshift & $95 \%$ & $68 \%$ & $68 \%$ & $95 \%$ \\
\hline 6 & 50.72 & 50.81 & 51.04 & 51.11 \\
7 & 50.69 & 50.73 & 50.85 & 50.90 \\
8 & 50.52 & 50.60 & 50.71 & 50.74 \\
9 & 50.27 & 50.41 & 50.62 & 50.69 \\
10 & 50.01 & 50.21 & 50.56 & 50.66 \\
$11^{\mathrm{a}}$ & 49.75 & 50.00 & 50.49 & 50.64 \\
$12^{\mathrm{a}}$ & 49.51 & 49.80 & 50.43 & 50.61 \\
$13^{\mathrm{a}}$ & 49.24 & 49.60 & 50.36 & 50.59 \\
$14^{\mathrm{a}}$ & 48.99 & 49.39 & 50.29 & 50.57 \\
$15^{\mathrm{a}}$ & 48.74 & 49.18 & 50.23 & 50.55 \\
\hline
\end{tabular}

Notes. The results tabulated here are featured in the lower right panel of Figure 2 and make use of all five key observational constraints considered here (Table 1). These results are derived in the context of the simple two-parameter model described in Section 3.

${ }^{\text {a }}$ Results here more sensitive to $\tau$ 's measurements from Planck and functional form adopted in modeling the ionizing emissivity evolution.

emissivity, as it implied optical depths of $<0.046$ (in tension with the Planck results at $1.7 \sigma$ ) and also extrapolated to $z=4.75$ implied ionizing emissivities (i.e., $\log _{10} \dot{N}_{\text {ion }}$ $\left.\left[\mathrm{s}^{-1} \mathrm{Mpc}^{-3}\right] \sim 51.5\right)$ in excess of that measured by BB $13 .{ }^{8}$

In Figure 3, evolution in the ionizing emissivity is presented, allowing for different values of the clumping factor $C_{\mathrm{H}_{\text {II }}}$ and also supposing that the clumping factor evolves in redshift as $1+43 z^{-1.71}$ as found by Pawlik et al. (2009) in sophisticated hydrodynamical simulations. The primary impact of changes in the clumping factor is on the overall normalization of ionizing emissivity $\dot{N}_{\text {ion }}$, not its evolution with time. This can be clearly seen in the maximum likelihood $\log _{10} \dot{N}_{\text {ion }}(z=8)$ and $\left(d \log _{10} \dot{N}_{\text {ion }} / d z\right)_{z=8}$ values presented in Table 3 for different values for the clumping parameter. Interestingly, the difference between the derived evolution of the ionizing emissivity with $C_{\mathrm{H}_{\text {II }}}=5$ and $C_{\mathrm{H}_{\text {II }}}=2$ is just $\sim 30 \%$. This is small (e.g., not a factor of 2.5 as might be suggested by the ratio of clumping factors) because reionization appears to be a photon-starved process (Bolton \& Haehnelt 2007). The overall insensitivity of estimates of the ionizing emissivity to the clumping factor $C_{\mathrm{H} \text { II }}$ is noteworthy.

Our $68 \%$ and $95 \%$ likelihood constraints on the model parameters $\log _{10} \dot{N}_{\text {ion }}(z=8)$ and $\left(d \log _{10} \dot{N}_{\text {ion }} / d z\right)_{z=8}$ are also presented in Figure 4 based on optical depths measured from Planck, the $Q_{\mathrm{H} \text { II }}$ 's estimated from the prevalence of Ly $\alpha$ in $z \sim$ 7-8 galaxies, and requiring that the model emissivity extrapolated to $z \sim 4.75$ matches that derived by BB13. A flat prior is assumed in deriving these constraints. The purple lines bracket the region allowed for reionization to be completed between $z=5.9$ and $z=6.5$.

\footnotetext{
8 The range of different constraints on $Q_{\mathrm{H} \text { II }}$ based on the prevalence of Ly $\alpha$ emission in galaxies (often using substantially identical observations) illustrate the challenges in deriving these $Q_{\mathrm{H} \text { II }}$ factors, as well as their considerable dependence on the simulations used to interpret the available observations (and indeed it is clearly non-trivial to adequately capture the many relevant physical phenomena that enter into these calculations, e.g., growth of structure, star formation, radiative transfer of $\operatorname{Ly} \alpha$ photons, and patchy reionization in the same simulation).
} 


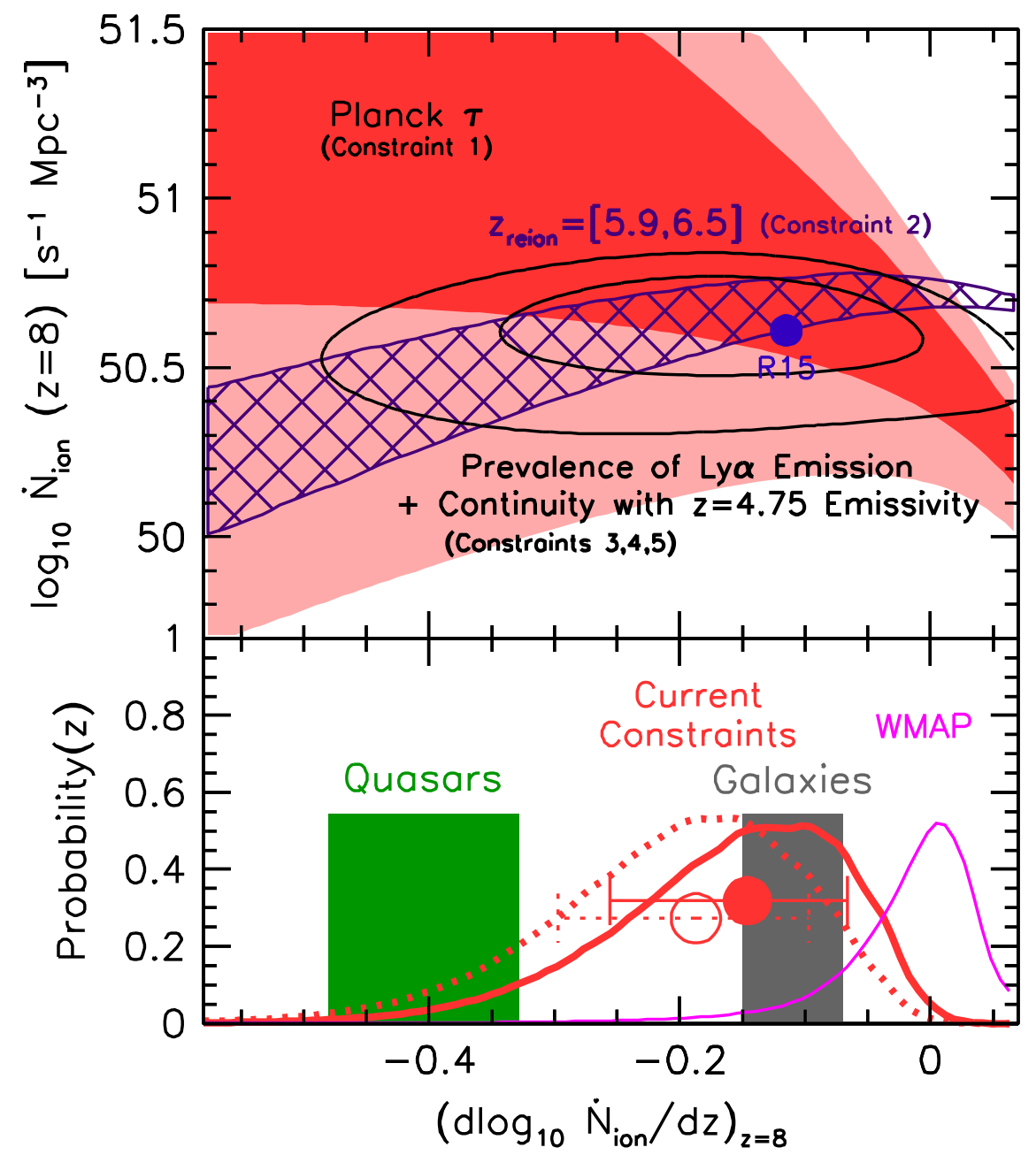

Figure 4. (Upper) Observational constraints on the ionizing emissivity $\dot{N}_{\text {ion }}(z=8)$ at $z \sim 8$ and the evolution in this emissivity per unit redshift $\left(d \log { }_{10} \dot{N}_{\text {ion }} / d z\right)_{z}=8$ (for $C_{\mathrm{H} \text { п }}=3$ ). $68 \%$ and $95 \%$ confidence intervals based on the Planck Thomson optical-depth constraints (red and light-red-shaded regions) and combining the prevalence of Ly $\alpha$ emission in $z \sim 7-8$ galaxies as found by S14 and continuity with the ionizing emissivity at $z=4.75$ as derived by Becker \& Bolton (2013: black lines). The purple lines bracket the allowed parameters assuming reionization is completed between $z=5.9$ and $z=6.5$ (i.e., when $Q_{\mathrm{H} \text { п }}$ reaches 1 using Equation (2)). The blue circle gives the equivalent evolution in the ionizing emissivity from the R15 models. (lower) The relative likelihood of different rates of evolution in the emissivity, $\left(d \log _{10} \dot{N}_{\text {ion }} / d z\right)_{z=8}$, derived by marginalizing over $\dot{N}_{\text {ion }}(z=8)$, along with the maximum-likelihood value and $1 \sigma$ uncertainties (red circle and $1 \sigma$ uncertainties). The dotted red line and open circle show the equivalent likelihoods, for a flat prior in $\dot{N}_{\text {ion }}(z=8)$ and $\left(d \log _{10} \dot{N}_{\text {ion }} / d z\right)_{z=8}(\operatorname{rather}$ than in $\tau$ : Section 4.1), respectively. Also presented (magenta line) is the equivalent likelihood using the WMAP optical depths and assuming reionization finishes between $z=5.9$ and $z=6.5$ (as in the upper left panel of Figure 2). The expected redshift dependence of the ionizing emissivity for galaxies and quasars is also shown with the shaded gray and green regions, respectively.

Marginalizing over all values of $\dot{N}_{\text {ion }}(z=8)$, we find a best estimate $\left(d \log _{10} \dot{N}_{\text {ion }} / d z\right)_{z=8}$ of $-0.15_{-0.11}^{+0.08}$ (red circle from the lower panel of Figure 4) based on the observed constraints. If we adopt a flat prior in $\left(d \log _{10} \dot{N}_{\text {ion }} / d z\right)_{z=8}$ and $\dot{N}_{\text {ion }}(z=8)$ (instead of our fiducial procedure of taking the prior to be flat in $\tau)$, we find a best estimate $\left(d \log _{10} \dot{N}_{\text {ion }} / d z\right)_{z=8}$ of $-0.19_{-0.11}^{+0.09}$ (open red circle from the lower panel of Figure 4).

It is worth emphasizing that the evolution we derive for the ionizing emissivity $\left(d \log _{10} \dot{N}_{\text {ion }} / d z\right)_{z=8}$ is only moderately affected by our use of constraints that depend on the prevalence of Ly $\alpha$ in $z \sim 7$ and $z \sim 8$ galaxies (constraints 3-4 in Table 1). Excluding these constraints, we find a best value for $\left(d \log _{10} \dot{N}_{\text {ion }} / d z\right)_{z=8}$ of $-0.09_{-0.13}^{+0.06}, \lesssim 0.5 \sigma$ different from our fiducial determination. The left panel of Figure 11 from Appendix B explicitly illustrates the evolution in the emissivity we would infer doing the analysis in this manner.

\subsection{Galaxies as the Primary Source of the Cosmic Ionizing Emissivity?}

We now consider whether galaxies could plausibly be the primary source of the inferred cosmic ionizing emissivity. To explore this question, we want to compare both the evolution of the ionizing emissivity and the evolution of the galaxy UVluminosity density.

To do this, we first examine the observed UV luminosity density from the B15 luminosity function (LF) study to observed magnitude limit -17 mag from current observations (see Figures 15, 18 and 19 from B15). The left panel of Figure 5 shows the observed results for the UV luminosity density $\rho_{\mathrm{UV}}$ from B15 and from other sources (e.g., Oesch et al. 2015; Ishigaki et al. 2015).

It is clear given the steep slope at $-17 \mathrm{mag}$ and the lack of any indication of a cut-off from Figures 15 and 19 in B15 for 
Table 3

Parameterization for the Cosmic Ionizing Emissivity Satisfying the Key Observational Constraints Considered Here

\begin{tabular}{|c|c|c|}
\hline$C_{\mathrm{H}_{\text {п }}}$ & $\begin{array}{c}\log _{10} \dot{N}_{\text {ion }}(z=8) \\
\left(\mathrm{s}^{-1} \mathrm{Mpc}^{-3}\right)\end{array}$ & $\overline{\left(d \log _{10} \dot{N}_{\text {ion }} / d z\right)_{z=8}}$ \\
\hline 2 & $50.62_{-0.07}^{+0.05}$ & $-0.15_{-0.11}^{+0.08}$ \\
\hline 3 (fiducial) $^{\mathrm{a}}$ & $50.67_{-0.08}^{+0.05}$ & $-0.15_{-0.11}^{+0.08}$ \\
\hline 3 (flat prior $\left.^{\mathrm{b}}\right)^{\mathrm{a}}$ & $50.65_{-0.09}^{+0.06}$ & $-0.19_{-0.11}^{+0.09}$ \\
\hline 5 & $50.75_{-0.09}^{+0.06}$ & $-0.14_{-0.10}^{+0.08}$ \\
\hline $1+43 z^{-1.71 \mathrm{c}}$ & $50.63_{-0.07}^{+0.05}$ & $-0.16_{-0.10}^{+0.07}$ \\
\hline \multicolumn{3}{|c|}{$\begin{array}{l}\text { Excluding Ly } \alpha \text { Prevalence-Type Constraints } \\
\text { (i.e., Excluding Constraints } 3-4 \text { from Table } 1 \text { ) }\end{array}$} \\
\hline 3 & $50.69_{-0.09}^{+0.05}$ & $-0.09_{-0.13}^{+0.06}$ \\
\hline \multicolumn{3}{|c|}{ More Highly Ionized Universe at $z>7$ than $\mathrm{S} 14^{\mathrm{e}}$} \\
\hline 3 & $50.72_{-0.03}^{+0.04}$ & $-0.05_{-0.04}^{+0.03}$ \\
\hline \multicolumn{3}{|c|}{ Less Highly Ionized Universe at $z \sim 7$ than $\mathrm{S} 14^{\mathrm{f}}$} \\
\hline 3 & $50.61_{-0.11}^{+0.07}$ & $-0.20_{-0.12}^{+0.10}$ \\
\hline \multicolumn{3}{|c|}{$W M A P \tau=0.089 \pm 0.014$, Reionization at $z=5.9-6.5$} \\
\hline 3 & $50.71_{-0.05}^{+0.04}$ & $0.00_{-0.06}^{+0.03}$ \\
\hline
\end{tabular}

Notes.

${ }^{a}$ For comparison, we note that only using the Planck $\tau=0.066 \pm 0.013$ optical depth constraint (PC15), requiring that reionization end at $z=5.9-6.5$, and assuming that $\log _{10} \dot{N}_{\text {ion }}(z=4.75)=50.99 \pm 0.45$ (BB13), we find $\log _{10} \dot{N}_{\text {ion }}(z=8)=50.66_{-0.15}^{+0.07}$ and $\left(d \log _{10} \dot{N}_{\text {ion }} / d z\right)_{z=8}=-0.16_{-0.15}^{+0.10} \quad$ for $C_{\mathrm{H} \text { II }}=3$.

${ }^{\mathrm{b}}$ In our fiducial determinations, the regions of parameter space are weighted according to $|\nabla \tau|$ (where the derivatives are with respect to $\log _{10} \dot{N}_{\text {ion }}$ and $\left.\left(d \log _{10} \dot{N}_{\text {ion }} / d z\right)_{z=8}\right)$. Hence, the prior is flat in units of optical depth $\tau$.

${ }^{\mathrm{c}}$ Redshift dependence found in the hydrodynamical simulations of Pawlik et al. (2009).

${ }^{\mathrm{d}}$ As illustrated in Appendix B and Figure 11.

${ }^{\mathrm{e}} Q_{\mathrm{H} \mathrm{II}}(z=7)=0.70 \pm 0.15, Q_{\mathrm{H} \text { II }}(z=8)=0.55 \pm 0.20$. Shown in the lowerleft panel of Figure 2 with the green-shaded region.

${ }^{\mathrm{f}} Q_{\mathrm{H} \mathrm{II}}(z=7)=0.49 \pm 0.15, Q_{\mathrm{H} \mathrm{II}}(z=8)=0.15 \pm 0.20$. Shown in the lowerleft panel of Figure 2 with the orange-shaded region.

example (see also McLure et al. 2013; Schenker et al. 2013; Alavi et al. 2014; Barone-Nugent et al. 2015), as well as early results from magnified sources found in lensing clusters (e.g., Atek et al. 2015) that fainter galaxies must contribute substantially to the total UV radiation from galaxies (e.g., Yan \& Windhorst 2004; Beckwith et al. 2006; Bouwens et al. 2007; Reddy \& Steidel 2009). While we can make plausible extrapolations based on the derived Schechter parameters, the question arises as to the appropriate limit.

As others have done (e.g., R13, R15) we adopt a fiducial luminosity of $-13 \mathrm{mag}$ down to which galaxies are typically assumed to be able to form efficiently. Faintward of $-13 \mathrm{mag}$, galaxy formation may be suppressed due to the inefficient gas cooling onto low-mass halos (e.g., Rees \& Ostriker 1977) or due to the difficulties in low-mass galaxies retaining their gas (e.g., Mac Low \& Ferrara 1999; Dijkstra et al. 2004). The choice of the limiting luminosity to adopt for the LF, e.g., $M_{\text {lim }}$, is also motivated from sophisticated hydrodynamical simulations (e.g., O'Shea et al. 2015) or from fits to the faintest points in the LF (e.g., Muñoz \& Loeb 2011; Barone-Nugent et al. 2015). Some guidance can also be obtained by attempting to match the redshift dependence inferred for ionizing emissivity $\dot{N}_{\text {ion }}(z)$ with $\rho_{\text {Uv }}$ (e.g., Bouwens et al. 2012a; R13), i.e., compare the inferred $\left(d \log _{10} \dot{N}_{\text {ion }} / d z\right)_{z=8}$ 's in Table 3
Table 4

UV Luminosity Density Derived from the B15 LF parameters Integrated to Different Faint-end Cut-offs $M_{\text {lim }}$ to the UV LF

\begin{tabular}{lc}
\hline \hline$M_{\lim }$ & $\log _{10} \rho_{\mathrm{UV}}\left(\mathrm{erg} \mathrm{s}^{-1} \mathrm{~Hz}^{-1} \mathrm{Mpc}^{-3}\right)$ \\
\hline-10 & $(26.47 \pm 0.08)-(0.07 \pm 0.05)(z-6)$ \\
-13 (fiducial) & $(26.37 \pm 0.05)-(0.11 \pm 0.04)(z-6)$ \\
-16 & $(26.20 \pm 0.03)-(0.16 \pm 0.03)(z-6)$ \\
$-17^{\mathrm{a}}$ & $(26.11 \pm 0.03)-(0.19 \pm 0.02)(z-6)$ \\
$-17.7^{\mathrm{a}}$ & $(26.02 \pm 0.03)-(0.21 \pm 0.02)(z-6)$ \\
\hline
\end{tabular}

Note.

${ }^{\mathrm{a}}$ The evolution of the UV luminosity density to -17 and -17.7 mag (as used by Bouwens et al. 2011; Ellis et al. 2013; Oesch et al. 2013, 2014, 2015; McLeod et al. 2015) is included for contrast with the evolution seen to much fainter limits and also for completeness. The steep slope of the UV LF at the -17 mag limit of deep searches for faint galaxies at $z \sim 7-8$ suggests that the cut-off is fainter than -17 mag (e.g., B15; McLure et al. 2013; Schenker et al. 2013). Recent results from $z \sim 7$ and $z \sim 8$ LFs derived from magnified sources behind lensing clusters also indicate a steep slope, and extends the limit at $z \sim$ 7 to -15.5 mag (e.g., Atek et al. 2015).

with the $d \rho_{\mathrm{UV}} / d z$ 's in Table 4 , but this presupposes that galaxies are the only source of the ionizing UV radiation and so it is considered indicative.

With our adopted limiting magnitude of $-13 \mathrm{mag}$, we estimate the UV-luminosity density $\rho_{\mathrm{UV}}$ implied by the B15 LF results, by marginalizing over the full likelihood distribution in $M^{*}, \phi^{*}$, and $\alpha$ and computing both the mean and $1 \sigma$ error from the resultant likelihood distribution for $\rho_{\mathrm{UV}}$ (similar to Figure 3 from R13).

The estimated UV-luminosity densities $\rho_{\mathrm{UV}}$ to -13 mag are shown in the right panel of Figure 5. The shallower redshift dependence of the result is due to the inclusion of much lowerluminosity galaxies in the calculated luminosity densities and the strong evidence that the UV luminosity density evolves faster at the bright end than the faint end (e.g., Bouwens et al. 2006, 2008; Yoshida et al. 2006; B15). The most important factor in this differential evolution is the flattening of the faintend slope $\alpha$ to the UV LF with cosmic time (e.g., Bouwens et al. 2011; McLure et al. 2013; Schenker et al. 2013). For context, the luminosity densities $\rho_{\mathrm{UV}}$ implied by the B15 Schechter parameters to many different limiting luminosities and as a function of redshift are presented in Table 4. The red regions on the two panels of Figure 5 will be discussed below following the discussion of Figure 6.

We are now positioned to explore both the evolution of the ionizing emissivity and the evolution of the galaxy UVluminosity density, as shown in the left and right panels of Figure 6, respectively, with the vertical axes chosen so that the inferred ionizing emissivity and luminosity density maximally overlap, i.e., $\dot{N}_{\text {ion }}(z)=f_{\text {esc }} \xi_{\text {ion }} \rho_{\text {UV }}$.

As is apparent from Figure 6, the best-fit evolution in the ionizing emissivity $\left(d \log _{10} \dot{N}_{\text {ion }} / d z\right)_{z=8}$ of $-0.15_{-0.11}^{+0.08}$ (or $-0.19_{-0.11}^{+0.09}$ adopting a flat prior in $\left(d \log _{10} \dot{N}_{\text {ion }} / d z\right)_{z=8}$ and $\left.\log _{10} \dot{N}_{\text {ion }}(z=8)\right)$ is in excellent agreement with the best-fit evolution in the UV luminosity density to $-13 \mathrm{mag}$, i.e., $d \log _{10} \rho_{\mathrm{UV}} / d z=-0.11 \pm 0.04$. While the uncertainties are still large, this is suggestive that early star-forming galaxies provide the ionizing photons needed to reionize the universe.

We emphasize that the present conclusions do not significantly depend on our use of the observational constraints 


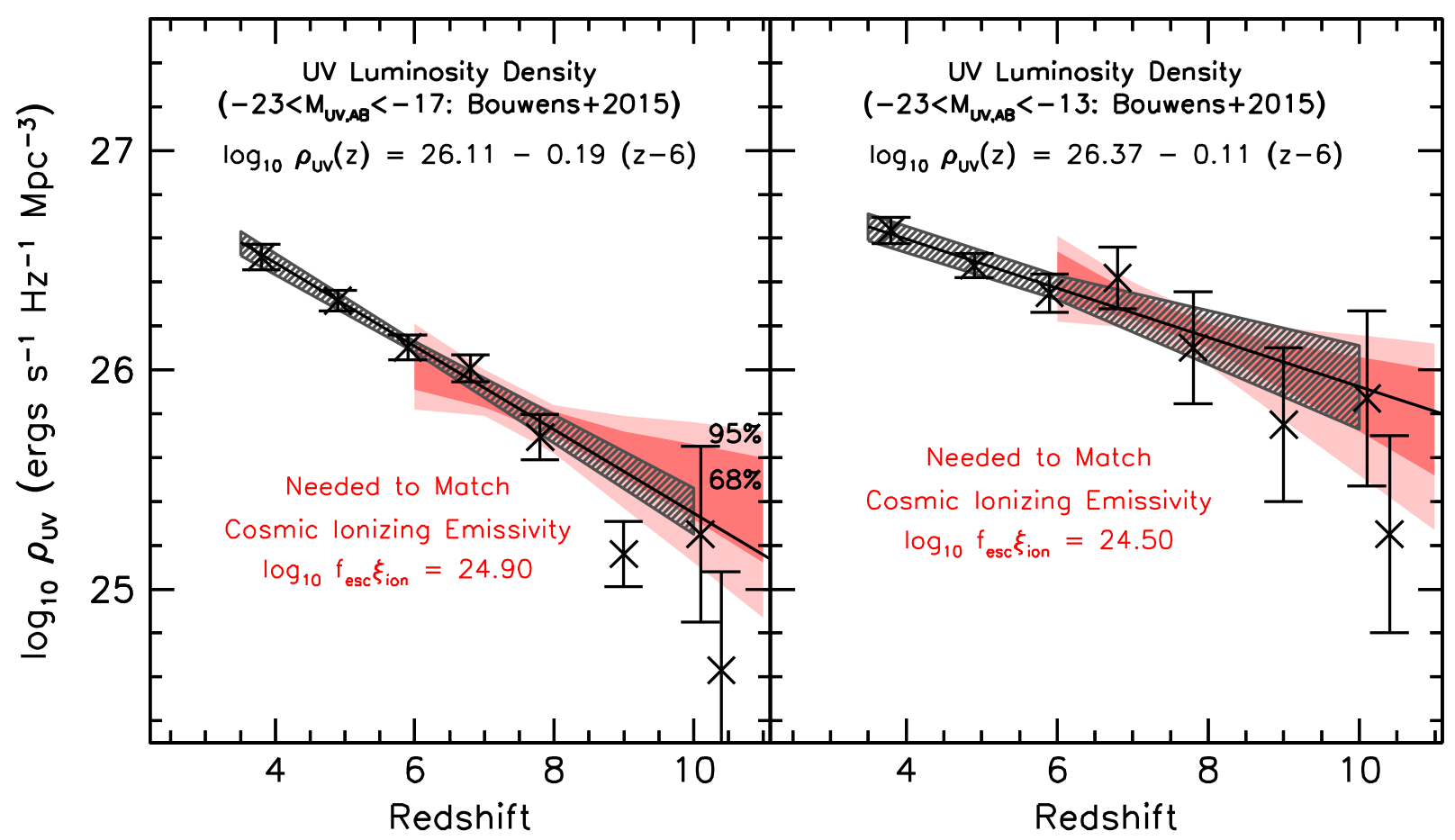

Figure 5. (Left) $68 \%$ and 95\% Confidence intervals (red and light red-shaded regions) on the UV luminosity density over the magnitude interval $-23<M_{\mathrm{UV}, \mathrm{AB}}<-17$ as derived by B15 in specific magnitude intervals (black crosses with $1 \sigma$ errors) and based on a fit to the Schechter parameters (black-hatched region). These constraints on the $z>6$ luminosity densities are supplemented by the $z \sim 9$ and $z \sim 10$ determinations by Ishigaki et al. (2015) and Oesch et al. (2014b), respectively. Also shown in the panel are the ionizing emissivities we infer here $\left(C_{\mathrm{H} \text { ॥ }}=3\right)$, offset by a redshift independent conversion factor $\log _{10} f_{\text {esc }} \xi_{\text {ion }}=24.95$. Even though it seems quite clear that UV LF extends faintward of -17 mag (e.g., Alavi et al. 2014; Atek et al. 2015; B15), we include this comparison here to illustrate the similar evolution observed when minimal extrapolations are employed. (Right) Same as left panel, but to a faint-end limit of -13 mag and adopting a conversion factor of $\log _{10} f_{\text {esc }} \xi_{\text {ion }}=24.50$. The UV luminosity density integrated down to -13 mag likely evolves more slowly with redshift than to $-17 \mathrm{mag}$, based on the steeper shape of the UV LF at high redshift due to an evolution to $\alpha$ (e.g., Bouwens et al. 2011; McLure et al. 2013) and possibly $M_{\mathrm{UV}}^{*}$ (e.g., Bowler et al. 2015; B15). The UV luminosity density integrated to $-13 \mathrm{mag}$ is also more uncertain than integrated to -17 mag due to the greater extrapolation required. In both the left and right panels, the UV luminosity density grows at a similar rate to the inferred ionizing emissivities (Section 4.2).

based on the prevalence of $\operatorname{Ly} \alpha$ emission from $z \sim 7$ and $z \sim 8$ galaxies. As we discuss in Section 4.1, if we exclude these constraints, the $\left(d \log _{10} \dot{N}_{\text {ion }} / d z\right)_{z=8} \quad$ value we derive $\left(-0.09_{-0.13}^{+0.06}\right)$ is almost identical to the best-fit evolution we find in the UV luminosity density. See Appendix B and Figure 11 where this point is illustrated explicitly.

It is interesting also to consider the offset where the ionizing emissivity and UV luminosity density maximally overlap. The best overlap occurs adopting an offset of $10^{24.50} \mathrm{~s}^{-1} /$ $\left(\mathrm{erg} \mathrm{s}^{-1} \mathrm{~Hz}^{-1}\right)\left(=f_{\text {esc }} \xi_{\text {ion }}\right)$ for a faint-end limiting luminosity of $-13 \mathrm{mag}$ (similar to what has been proposed in many other studies: e.g., KF12, R13). This conversion factor has an uncertainty of at least 0.10 dex, given the uncertainties on both the normalization of the ionizing emissivity at $z \sim 8$ (typically $\sim 0.08$ dex: Table 3 ) and the $\gtrsim 0.05$ dex uncertainties on the UV luminosity density integrated to $\lesssim-13$ mag (see Table 4 ). This is one of the first analyses to estimate an uncertainty on this conversion factor using observations that directly concern the $z>6$ universe (see also Mitra et al. 2013). The evolution of the ionizing emissivity is also shown in relation to the $\rho_{\mathrm{UV}}$ for the two magnitude limits in Figure 5, using an appropriate offset. It is quite clear that the two quantities seem to evolve in a similar manner even to the bright limit.

Is this multiplicative offset plausible if star-forming galaxies are to be the source of the cosmic ionizing emissivity? Given that it can be expressed as the product of two factors $\xi_{\text {ion }}$, the production efficiency of Lyman-continuum photons per unit UV luminosity, and the escape fraction $f_{\text {esc }}$ with plausible values in the range $10^{25.2}-10^{25.5} \mathrm{~s}^{-1} /\left(\mathrm{erg} \mathrm{s}^{-1} \mathrm{~Hz}^{-1}\right)$ and $0.05-0.40$ based on observations of $z \sim 2-4$ galaxies (Vanzella et al. 2012; Mostardi et al. 2013; R13), the observed offset between the inferred ionizing emissivity and UV luminosity density is certainly within the range allowed by these value, i.e., $10^{23.9}-10^{25.1} \mathrm{~s}^{-1} /\left(\mathrm{erg} \mathrm{s}^{-1} \mathrm{~Hz}^{-1}\right)$. This is the case for all three limiting luminosities $M_{\text {lim }}$ presented in Figure 7 ( $-10 \mathrm{mag},-13 \mathrm{mag}$, and $-16 \mathrm{mag})$.

In distinct contrast to the situation faced in interpreting the $W M A P \tau$ 's, no evolution in the escape fraction is required to match the ionizing emissivity model we infer, as had been earlier considered by HM12 or KF12. Neither is evolution in the UV-continuum slopes $\beta$ required, despite the apparent trend toward bluer $\beta$ 's at high redshift (e.g., $d \beta / d z \sim-0.10 \pm 0.05$ : Bouwens et al. 2014: see also Wilkins et al. 2011; Bouwens et al. 2012b; Finkelstein et al. 2012b; Kurczynski et al. 2014) or as expected from simple theoretical models (e.g., $d \beta / d z \sim$ -0.04 [Wilkins et al. 2013] or $d \beta / d z \sim-0.1$ [Finlator et al. 2011]). A mild evolution in $\beta$ is not inconsistent with our constraints on the ionizing emissivity.

Given the likely connection between the ionizing emissivities we infer and galaxy UV luminosity density at $z>6$, what are physically plausible values for $\xi_{\text {ion }}$ and $f_{\text {esc }}$ that we would infer? The relevant value of the UV-continuum slopes $\beta$ of galaxies to estimate their ionizing emissivity contribution is $\sim-2.3$, as most of the luminosity density at $z \geqslant 6$ occurs in lower luminosity galaxies and the median $\beta$ for faint $z \sim 7-8$ galaxies is $\sim-2.3$ (e.g., Bouwens et al. 2014). Using a similar 

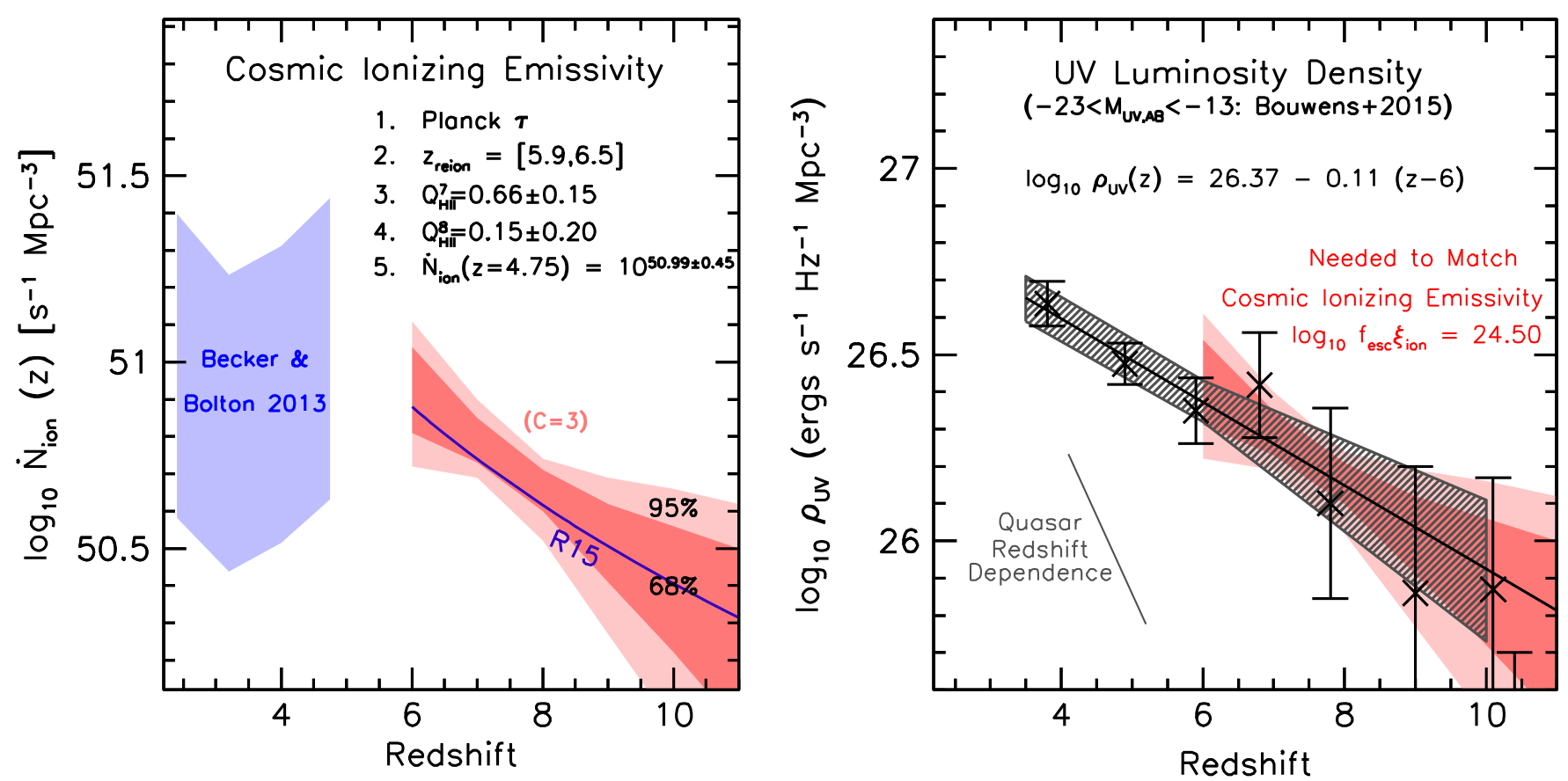

Figure 6. (Left) $68 \%$ and $95 \%$ confidence intervals (red and light red-shaded regions) on the evolution of the cosmic ionizing emissivity (assuming $C_{\mathrm{H} \text { II }}=3$ ). The derived evolution presented is the same as in the lower right panel of Figure 2 (see also Table 2 for these constraints in tabular form). Constraints from BB13 are indicated with the light-blue-shaded region. The equivalent trend in $\dot{N}_{\text {ion }}$ derived from R15's model (blue line) is shown here for context and is included among those ionizing emissivity evolution models preferred at $68 \%$ confidence. (Right) $68 \%$ confidence intervals on the UV-luminosity density over the magnitude interval $-23<M_{\mathrm{UV}, \mathrm{AB}}<-13$ as derived by B15 in specific redshift intervals (black crosses with $1 \sigma$ errors). See Table 4 and Figure 5 for the calculated luminosity densities to other faint-end limits. These constraints on the $z>6$ luminosity densities are supplemented by the $z \sim 9$ and $z \sim 10$ determinations by Ishigaki et al. (2015) and Oesch et al. (2014b), respectively, extrapolated to the same limiting luminosity ( $-13 \mathrm{mag}$ ). The black-hashed region shows the implied evolution of the galaxy UVluminosity density, based on our constraints on the ionizing emissivity and assuming that galaxies are the source of this emissivity with some redshift-independent conversion factor $\log _{10} f_{\text {esc }} \xi_{\text {ion }}=24.50$. The steep gray line shows the redshift dependence one would expect (with approximately the correct normalization [in units of $\left.\dot{N}_{\text {ion }}\right]$ relative to other quantities presented here) for the ionizing emissivity for quasars using the LEDE-model fit from McGreer et al. (2013: see Section 4.3). The UV luminosity density and ionizing emissivity we infer appear to evolve very similarly (Section 4.2).

approach to R13, one can show that $\xi_{\text {ion }}$ in such galaxies has an approximate value of $10^{25.46} \mathrm{~s}^{-1} /\left(\mathrm{erg} \mathrm{s}^{-1} \mathrm{~Hz}^{-1}\right)$ for $\xi_{\text {ion }}$ (Appendix A), similar to the $\xi_{\text {ion }}$ advocated by Topping \& Shull (2015). For this value of $\xi_{\text {ion }}$, the escape fraction $f_{\text {esc }}$ is $0.11_{-0.02}^{+0.03}$ for the observed conversion factor $10^{24.50} \mathrm{~s}^{-1} /$ $\left(\operatorname{erg~s}^{-1} \mathrm{~Hz}^{-1}\right)$. Alternatively, if we take $\xi_{\text {ion }}$ to be equal to $10^{25.2} \mathrm{~s}^{-1} /\left(\mathrm{erg} \mathrm{s}^{-1} \mathrm{~Hz}^{-1}\right)$ as R13 adopted to match with the $\beta$ measurements of Dunlop et al. (2013), the relevant value of $f_{\text {esc }}$ is $0.20_{-0.04}^{+0.05}$ (essentially identical to R13's adopted value of 0.2 ).

Clearly, many degenerate combinations of $f_{\text {esc }}, \xi_{\text {ion }}$, and $M_{\text {lim }}$ can be successful in producing the same ionizing emissivity. For variable $M_{\mathrm{lim}}$, the present constraint on $f_{\mathrm{esc}} \xi_{\text {ion }}$ can be generalized to the following constraint on these three parameters:

$$
f_{\text {esc }} \xi_{\text {ion }} f_{\text {corr }}\left(M_{\text {lim }}\right)=10^{24.50} \mathrm{~s}^{-1} /\left(\mathrm{erg} \mathrm{s}^{-1} \mathrm{~Hz}^{-1}\right)
$$

where the added term $f_{\text {corr }}\left(M_{\text {lim }}\right)=$ $10^{0.02+0.078\left(M_{\text {lim }}+13\right)-0.0088\left(M_{\text {lim }}+13\right)^{2}}$ corrects $\rho_{\mathrm{UV}}(z=8)$ derived to a faint-end limit of $M_{\lim }=-13$ mag to account for different $M_{\text {lim }}$ 's. ${ }^{9}$ For clumping factors $C_{\mathrm{H} \text { II }}$ not equal to our fiducial value of 3 , the left-hand side of the above equation should be

\footnotetext{
9 In deriving this correction factor, we made use of the following relationship between $\rho_{\mathrm{UV}}(z=8)$ and the faint-end cut-off $M_{\lim }$ to the LF: $\log _{10} \rho_{\mathrm{UV}}(z=8)$ $=(26.17 \pm 0.09)+(0.08 \pm 0.02)\left(M_{\mathrm{lim}}+13\right)-(0.009 \pm 0.008)$

$\left(M_{\lim }+13\right)^{2}$. This relationship can be derived by fitting to the results in Table 4 and is accurate to $5 \%$.
}

multiplied by $\left(C_{\mathrm{H} \text { II }} / 3\right)^{-0.3}$ based on the results presented in Table 3 (the $\left(C_{\mathrm{H} \text { II }} / 3\right)^{-0.3}$ scaling is approximately valid for $\left.C_{\mathrm{H} \text { II }}<10\right)$. The factor $10^{24.50} \mathrm{~s}^{-1} /\left(\mathrm{erg} \mathrm{s}^{-1} \mathrm{~Hz}^{-1}\right)$ has an uncertainty of $\sim 0.1$ dex.

Equation (5) can be manipulated to allow for an estimate of $f_{\text {esc }}$ given assumed values for the other parameters $M_{\text {lim }}, \xi_{\text {ion }}$, and $C_{\mathrm{H} \text { II }}$, assuming that galaxies reionize the universe:

$$
f_{\mathrm{esc}} \approx \xi_{\text {ion }}^{-1} f_{\text {corr }}^{-1}\left(M_{\mathrm{lim}}\right)\left(C_{\mathrm{H} \mathrm{II}} / 3\right)^{0.3} 10^{24.50} \mathrm{~s}^{-1} /\left(\mathrm{erg} \mathrm{s}^{-1} \mathrm{~Hz}^{-1}\right) .
$$

Estimates of $f_{\text {esc }}$ for various fiducial choices of $\xi_{\text {ion }}, M_{\text {lim }}$, and clumping factors $C_{\mathrm{H} \text { п }}$ are provided in Table 5. This is one of the first analyses to estimate the uncertainty on the derived value of $f_{\text {esc }}$ based on observational constraints on the ionization state of the $z>6$ universe (see also Mitra et al. 2013).

The issue of degeneracies among the parameters $f_{\text {esc }}, \xi_{\text {ion }}$, and $M_{\text {lim }}$ is discussed extensively in KF12 (see also Bouwens et al. 2012a; Finkelstein et al. 2012a; R13) and may not be easy to resolve based on observations in the immediate future.

\subsection{Quasars as the Primary Source of the Cosmic Ionizing Emissivity?}

We briefly consider whether quasars could be the primary source of the cosmic ionizing emissivity. Despite their relative scarcity in the $z>4$ universe, quasars potentially can 


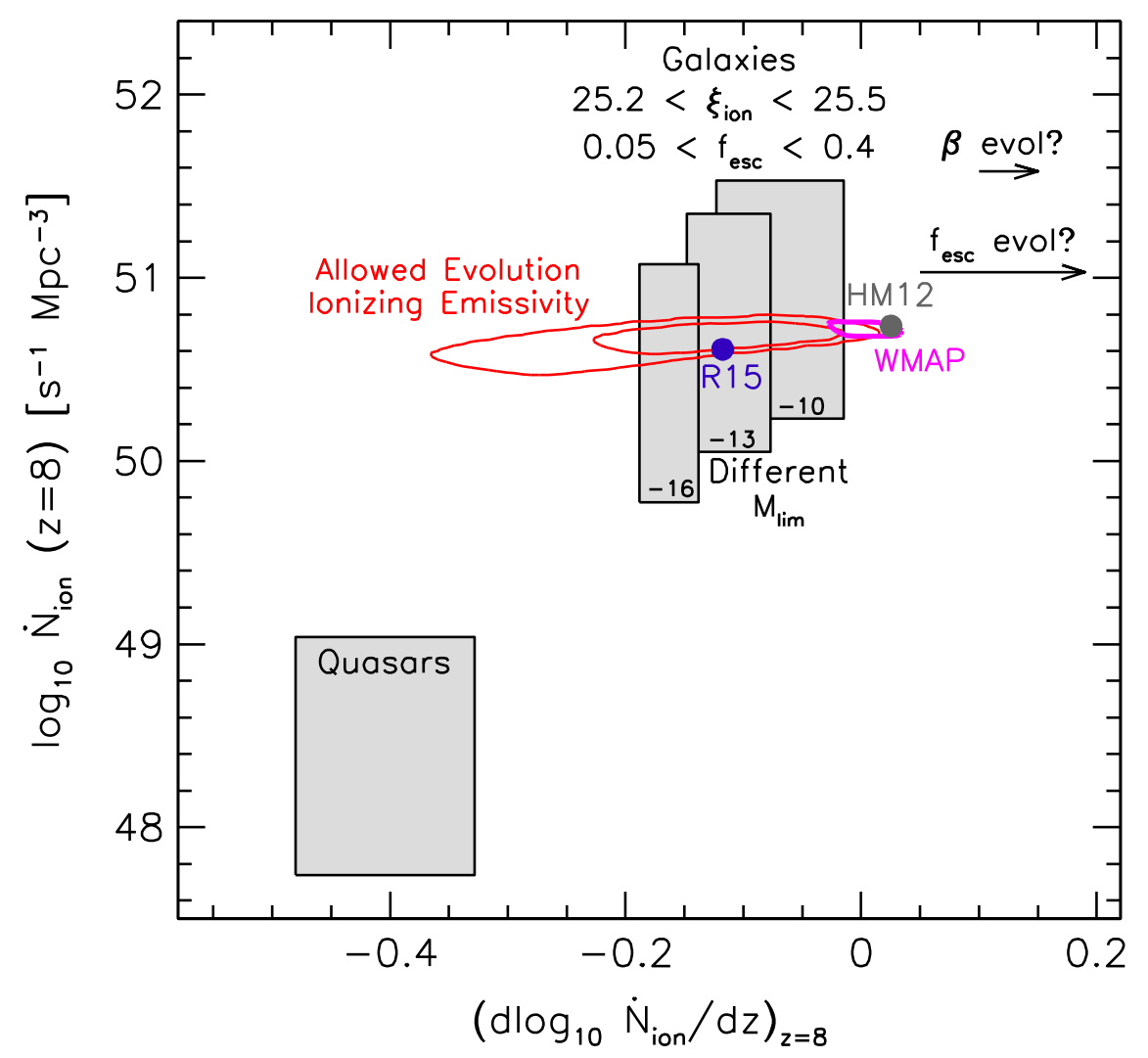

Figure 7. $68 \%$ and $95 \%$ confidence intervals on parameters describing the cosmic ionizing emissivity (assuming $C_{\mathrm{H} \text { I }}=3$ : red contours) and a comparison to the emissivity expected (gray boxes in the upper middle) for galaxies to three different faint-end cut-offs to the LF $(-10,-13$, and -16 mag) where the Lymancontinuum photon production efficiency $\xi_{\text {ion }}$ and $f_{\text {esc }}$ plausibly have values over the wide range: $10^{25.2}-10^{25.5} \mathrm{~s}^{-1} /\left(\mathrm{erg} \mathrm{s}^{-1} \mathrm{~Hz}^{-1}\right)$ and $0.05-0.40$ based on the observations (e.g., Siana et al. 2010, 2015; Vanzella et al. 2012; Mostardi et al. 2013; Bouwens et al. 2014; Cooke et al. 2014). The arrows indicate how potential evolution in the UV-continuum slopes $\beta$ 's or $f_{\text {esc }}$ of galaxies (as $d \beta / d z \sim-0.04$ [predicted by Wilkins et al. 2013 and consistent with the evolution observed by Bouwens et al. 2014] or $f_{\text {esc }} \propto(1+z)^{3.4}$ : required by HM12 to match the WMAP $\tau$ measurements) would affect the evolution of $\dot{N}_{\text {ion. }}$. The gray box to the lower left shows the expectations for quasars using the Willott et al. (2010) results to set the normalization of the ionizing emissivity $\dot{N}_{\text {ion }}(z)$ (after correcting their results upwards by 0.2 dex to account for a possibly steeper faint-end slope $\alpha \sim-2$ vs. the $\alpha=-1.5$ Willott et al. 2010 adopt) and the redshift dependence of the emissivity from the McGreer et al. (2013) LEDE and modified LEDE models. The 68\% likelihood contours preferred based on the WMAP opticle depths and assuming reionization finishes at $z=5.9-6.5$ are shown in magenta. The small size of the WMAP contours is an artifact of the sensitivity of the computed optical depths $\tau$ to small changes in $\left(d \log _{10} \dot{N}_{\text {ion }} / d z\right)_{z=8}$ when $\left(d \log _{10} \dot{N}_{\text {ion }} / d z\right)_{z=8}$ approaches 0 (since such implies a constant $\rho_{\text {UV }}$ to arbitrarily high redshift). The gray and blue circles give the equivalent parameters from the HM12 and R15 models, respectively. It is clear that galaxies should be successful at producing the inferred ionizing emissivity for a variety of plausible values for $f_{\text {esc }}, \xi_{\text {ion }}$, and $M_{\text {lim. }}$. It is also clear from this figure that quasars/AGN seem quite unlikely to be the source of this emissivity (see also HM12).

Table 5

Required Values of $f_{\text {esc }}$ for Different $M_{\text {lim }}, \xi_{\text {ion }}$, and Clumping Factors $C_{\mathrm{H} \mathrm{II}}$ Assuming that Galaxies Drive the Reionization of the Universe ${ }^{\mathrm{a}}$

\begin{tabular}{|c|c|c|c|c|c|c|}
\hline \multirow[b]{3}{*}{$C_{\mathrm{H} \text { п }}$} & \multicolumn{6}{|c|}{ Required $f_{\text {esc }}$} \\
\hline & \multicolumn{3}{|c|}{$\xi_{\text {ion }}=10^{25.46} \mathrm{~s}^{-1} /\left(\mathrm{erg} \mathrm{s}^{-1} \mathrm{~Hz}^{-1}\right)$} & \multicolumn{3}{|c|}{$\xi_{\text {ion }}=10^{25.2} \mathrm{~s}^{-1} /\left(\mathrm{erg} \mathrm{s}^{-1} \mathrm{~Hz}^{-1}\right)^{\mathrm{b}}$} \\
\hline & $M_{\lim }=-17$ & $M_{\lim }=-13$ & $M_{\mathrm{lim}}=-10$ & $M_{\lim }=-17$ & $M_{\lim }=-13$ & $M_{\lim }=-10$ \\
\hline 2 & $0.26_{-0.05}^{+0.07}$ & $0.10_{-0.02}^{+0.03}$ & $0.06_{-0.01}^{+0.02}$ & $0.46_{-0.10}^{+0.12}$ & $0.18_{-0.04}^{+0.05}$ & $0.12_{-0.02}^{+0.03}$ \\
\hline 3 & $0.29_{-0.06}^{+0.07}$ & $0.11_{-0.02}^{+0.03}$ & $0.07_{-0.01}^{+0.02}$ & $0.52_{-0.11}^{+0.14}$ & $0.20_{-0.04}^{+0.05 \mathrm{c}}$ & $0.13_{-0.03}^{+0.03}$ \\
\hline 5 & $0.34_{-0.07}^{+0.09}$ & $0.13_{-0.03}^{+0.03}$ & $0.08_{-0.02}^{+0.02}$ & $0.61_{-0.13}^{+0.16}$ & $0.23_{-0.05}^{+0.06}$ & $0.15_{-0.03}^{+0.04}$ \\
\hline $1+43 z^{-1.71^{d}}$ & $0.27_{-0.06}^{+0.07}$ & $0.10_{-0.02}^{+0.03}$ & $0.07_{-0.01}^{+0.02}$ & $0.49_{-0.10}^{+0.13}$ & $0.19_{-0.04}^{+0.05}$ & $0.12_{-0.03}^{+0.03}$ \\
\hline
\end{tabular}

Notes.

${ }^{\mathrm{a}}$ These $f_{\text {esc }}$ factors can be derived from Equation (6) in Section 4.2 of this paper. Importantly, we can also quote uncertainties on the estimated $f_{\text {esc }}$ 's, which follow from our $1 \sigma$ error estimate $\left(\sim 0.1\right.$ dex) on the conversion factor $10^{24.50} \mathrm{~s}^{-1} /\left(\mathrm{erg} \mathrm{s}^{-1} \mathrm{~Hz}^{-1}\right)$ from UV luminosity density $\rho_{\mathrm{UV}}$ to the equivalent ionizing emissivity $\dot{N}_{\text {ion }}$. Constraints on $f_{\text {esc }}$ are also attempted by KF12 at $z \sim 4$ based on the derived $\dot{N}_{\text {ion }}$ there (see also Finkelstein et al. 2012a for an estimated $f_{\text {esc }}$ at $z \sim 6$ based on $\dot{N}_{\text {ion }}$ from Bolton \& Haehnelt 2007).

b Adopted by R13.

${ }^{\mathrm{c}}$ In fact, this is the same $f_{\text {esc }}$ that R13 and R15 suggest using for the fiducial parameter choices they adopt: $\xi_{\text {ion }}=10^{25.2} \mathrm{~s}^{-1} /\left(\mathrm{erg} \mathrm{s}^{-1} \mathrm{~Hz}^{-1}\right), M_{\text {lim }}=-13$, and $C_{\mathrm{H} \text { II }}$ $=3$. However, no attempt was made by R15 to provide a constraint on the uncertainties in this estimate of $f_{\text {esc }}$ as derived here.

${ }^{\mathrm{d}}$ Redshift Dependence found in the hydrodynamical simulations of Pawlik et al. (2009). 
t (Gyr)

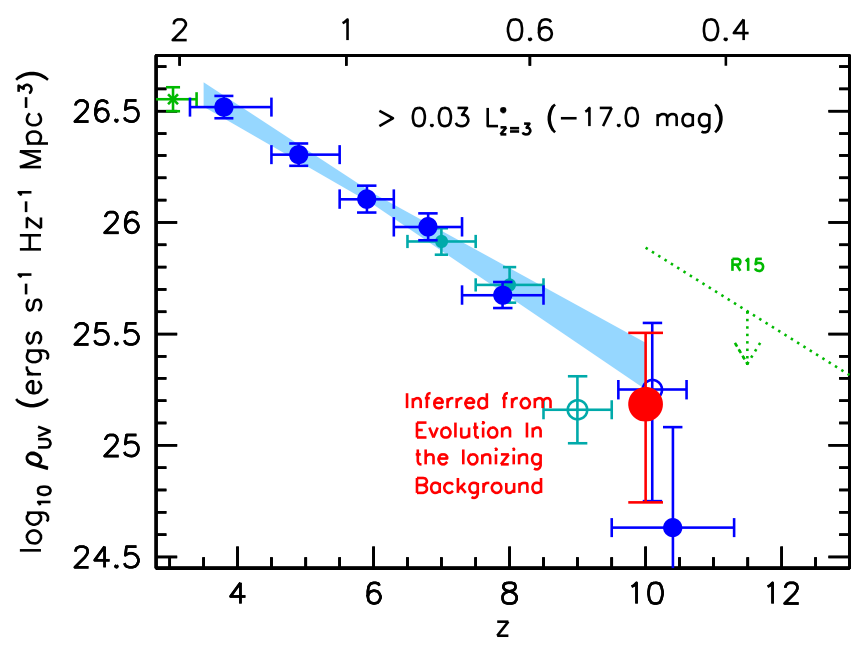

Figure 8. Constraints on the UV luminosity density at $z \sim 10$ to the typical observed limit of $-17 \mathrm{mag}$ (red circle), derived from the $z=6-10$ evolution of the ionizing emissivity (Section 4.4). The luminosity density results from B15 at $z=6$ are used as a baseline. The derived UV luminosity density at $z=10$ is also corrected downward by -0.32 dex to account for the different evolution in $\rho_{\mathrm{UV}}$ expected to $-17 \mathrm{mag}$ vs. to $-13 \mathrm{mag}$ (see Table 4 ). For context, estimates of the UV luminosity density to the same faint-end limit based on the Reddy \& Steidel (2009) $z=3$ results (green cross), the B15 $z=4-10$ results (blue circles), the McLure et al. (2013) $z=7-8$ results (cyan circles), the Ishigaki et al. (2015) $z=9$ (cyan open circles), and Oesch et al. (2014b) $z=10$ results (blue open circles) are also shown. The shaded-light-blue curve indicates the evolution in UV luminosity density one would expect based on extrapolations of the B15 LF parameters to $z>8$. The dotted green $1 \sigma$ upper limit at $z=11.5$ shows constraints on the UV luminosity density at $z>10$ from R15 derived using the recent Planck $\tau$ measurements corrected to -17 mag based on the empirical scalings presented in Table 4.

contribute quite substantially to the inferred emissivity due to the hardness of their spectrum and a much higher escape fraction $\left(f_{\text {esc }}=1\right.$ ?: e.g., Loeb \& Barkana 2001).

While a fraction of the emissivity from quasars at $z>4$ is expected to originate from the most luminous sources, a potentially large fraction of their contribution could originate at much lower luminosities, and therefore it is important to have reasonable constraints on both the volume densities of faint quasars (including active galactic nuclei; AGN) and their faintend slopes to effectively estimate the ionizing emissivity they produce.

Perhaps the deepest, wide-area probes of the $z>4$ quasar LFs are provided by Willott et al. $(2009,2010)$ and McGreer et al. (2013). The deepest part of Willott et al. (2009, 2010) searches for faint $(>-22 \mathrm{mag}) z \sim 6$ QSOs over $4.5 \mathrm{deg}^{2}$, while McGreer et al. (2013) leveraged the deep observations over a $235 \mathrm{deg}^{2}$ region in SDSS Stripe 82 to probe the prevalence of moderately faint $(>-24$ mag) $z \sim 5$ qusars. Willott et al. (2009) identified 1 very faint $z=6.01$ quasar over the CFHTLS Deep/SXDS, while McGreer et al. (2013) identified some $\sim 70$ faint quasars in their search, 29 with absolute magnitudes faintward of $-25 \mathrm{mag}$.

Given the relatively small numbers of faint quasars identified by these programs (and other recent searches e.g., Weigel et al. 2015, who find no convincing $z \gtrsim 5$ AGN over the Chandra Deep Field South), the total emissivity of ionizing photons from the quasar population at $z \sim 4-6$ is still quite uncertain. Nevertheless, a sufficient number of faint quasars have been found that estimates of the total emissivity of the population can still be made (but see the discussion in Giallongo et al. 2015). At this time, the most reliable estimates can be made using the faint-end slopes $\alpha$ and evolutionary trends derived by McGreer et al. (2013). McGreer et al. (2013) find a $\log _{10} \phi(z)=\log _{10} \phi_{0}-0.60(z-2.2)$ and $M_{*}(z)=$ $M_{*, 0}-0.68(z-2.2)$ trend with their LEDE model and a $\log _{10} \phi(z)=\log _{10} \phi_{0}-0.70(z-2.2)$ and $M_{*}(z)=M_{*, 0}-$ $0.55(z-2.2)$ trend with their modified LEDE model. These models imply a comoving emissivity $\log _{10} \epsilon(z)$ that evolves as $-0.33(z-2.2)$ and $-0.48(z-2.2)$.

If we take the ionizing emissivity estimates that Willott et al. (2010) derive from their deep $z \sim 6$ search, extrapolate to $z \sim 8$, and adjust their result upwards by 0.2 dex to account for the steeper faint-end slope of $\alpha=-2$ found at $z \sim 5$ by McGreer et al. (2013: versus an assumed faint-end slope of $\alpha=-1.5$ by Willott et al. 2010), the $\log _{10} \dot{N}_{\text {ion }}(z=8)$ we would derive is 48.5 , with a $\left(d \log _{10} \dot{N}_{\text {ion }} / d z\right)_{z=8}$ of $-0.41 \pm$ 0.08 taking the arithmetic mean of the two LEDE models considered by McGreer et al. (2013). Fan et al. (2001) derive $-0.47 \pm 0.15$ based on the evolution of the space density of bright quasars from $z=6$ to $z=3$. If we compare these parameters with the values we derive for the ionizing emissivity, it is clear that faint quasars do not appear to come close to providing enough photons to drive the reionization of the universe. In addition, the ionizing emissivity produced by this population shows a redshift dependence that is slightly steeper than what we require to reproduce the observational constraints (see e.g. the green-shaded region and the red line at the bottom of Figure 4).

Is it possible that even fainter quasars/AGN could boost these luminosity densities? Given that the faint-end slope $\alpha$ McGreer et al. (2013) find at $z \sim 5$ is $-2.03_{-0.14}^{+0.15}$, the luminosity density will be only logarithmically divergent, which for an integration to $\sim-22$ and $\sim-16$ mag (if such fainter quasars/ AGN exist in large numbers) would only increase the overall luminosity density by a factor of $1.5(0.2 \mathrm{dex})$ and a factor of 4 (0.6 dex). This suggests that a full consideration of the contribution from faint quasars can potentially boost the total ionizing emissivity produced by quasars. However, even with such steps, quasars appear quite unlikely to contribute meaningfully to the reionization of the universe at $z>6$ (but see Giallongo et al. 2015).

\subsection{Implications for the UV Luminosity Density at $z \sim 10$ ?}

The current discussion and others (e.g., R15; Mitra et al. 2015) suggest that star-forming galaxies produce the bulk of the ionizing emissivity. Given this, we can use current constraints on the evolution of the ionizing emissivity to provide an estimate of the UV luminosity density at $z \sim 10$. This is useful since there has been much discussion about whether the UV luminosity and SFR density are trending differently with time from $z \sim 10$ to $z \sim 8$ than at later times (e.g., Oesch et al. 2012, 2013, 2014, 2015; Ellis et al. 2013; McLeod et al. 2014; Coe et al. 2015; Ishigaki et al. 2015).

In the simplest case of no evolution in $f_{\text {esc }}$ or $\xi_{\text {ion }}$, evolution in the UV luminosity density would mirror that seen in ionizing emissivity. In this case, one would expect a factor of $10^{4}\left(-0.15_{-0.11}^{+0.08}\right)=4_{-2}^{+7}$ increase in the UV luminosity density 

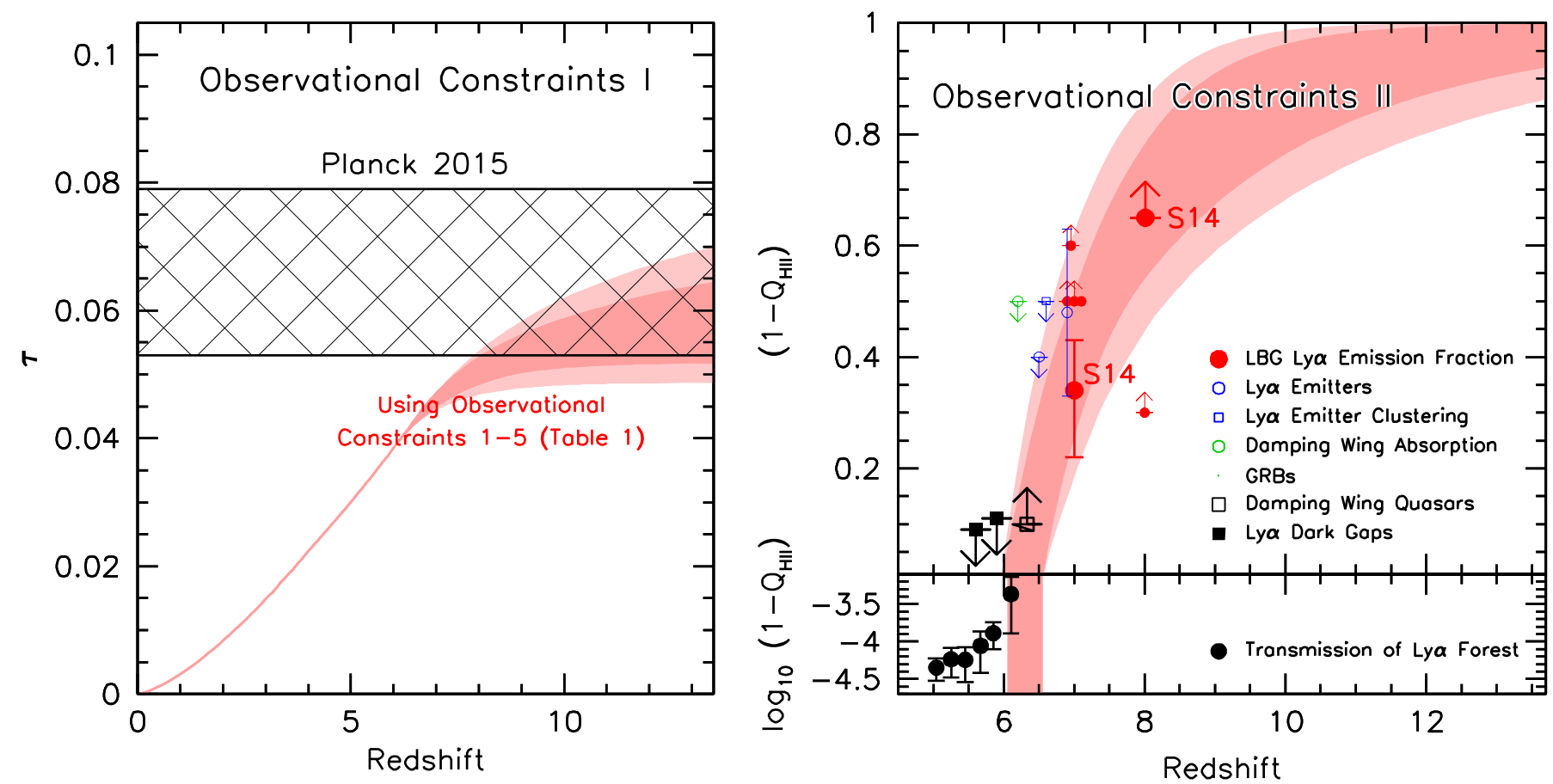

Figure 9. Comparison of the key observational constraints considered here with the results from the simple two-parameters models for the cosmic ionizing emissivity preferred at 68\% and 95\% confidence (Section 4.5). (Left) Shown are the constraints on the Thomson optical depth $\tau$ provided by the Planck three-year results (PC15: cross-hatched black region). The red and light-red-shaded regions show the range of cumulative Thomson optical depths for our models of the ionizing emissivity preferred at 68\% and 95\% confidence, respectively (Figure 4), and where reionization is complete between $z=5.9-6.5$. (Right) shown are constraints on the filling factor of ionized hydrogen $Q_{\mathrm{H} \text { II }}$ as a function of redshift. The constraints are largely as compiled by R15 (see Table 1 of the present manuscript) and are based on the Gunn-Peterson optical depths and dark-gap statistics measured in $z \sim 6$ quasars (Fan et al. 2006b; McGreer et al. 2015: solid black circles and squares), damping wings measured in $z \sim 6.2-6.4$ quasars (Schroeder et al. 2013: open black square) and a $z=6.3$ GRB (Totani et al. 2006; McQuinn et al. 2008: open green circle), Ly $\alpha$-emitter LFs and clustering statistics at $z \sim 6.6$ (Ouchi et al. 2010: open blue square and circle) and at $z \sim 7$ (Ota et al. 2008: open blue circle), and the prevalence of Ly $\alpha$ emission in $z \sim 7-8$ galaxies (S14: large red circles). Also included here (small red solid circles) are other estimated constraints on $Q_{\mathrm{H}}$ ) from the prevalence of Ly $\alpha$ emission from galaxies at $z \sim 7\left(Q_{\mathrm{H} \text { п }}<0.5\right.$ [R13]; $Q_{\mathrm{H} \text { п }}<0.4$ [Ono et al. 2012]; $Q_{\mathrm{H} \text { п }}<0.49$ [P14]; $Q_{\mathrm{H} \text { п }} \sim 0.5$ [Caruana et al. 2014]) and at $z \sim 8$ $\left(Q_{\mathrm{H} \text { п }}<0.7\right.$ [Tilvi et al. 2014]). The red and light-red-shaded region indicates the range of $Q_{\mathrm{H} \text { п }}$ allowed for our models of the ionizing emissivity preferred at $68 \%$ and $95 \%$ confidence, respectively, and where reionization is complete between $z=5.9$ and $z=6.5$. The magenta-hatched region indicates the range of $Q_{\mathrm{H} \text { п }}$ allowed at $68 \%$ confidence for the WMAP nine-year $\tau$ measurement $(0.089 \pm 0.014)$.

from $z \sim 10$ to $z \sim 6$ integrated to the faint-end cut-off to the LF $M_{\text {lim }}$, which we will take to be -13 mag. However, since the UV luminosity density is measured directly from observations to about $-17 \mathrm{AB}$ mag in the reionization epoch, it is perhaps preferable to evaluate the changes to that welldetermined limit and to correct the derived results from the ionizing emissivity to $-17 \mathrm{mag}$ instead of the extrapolated $-13 \mathrm{mag}$ limit. Considering the evolution integrated to $-17 \mathrm{mag}$, we would expect $d \log _{10} \rho_{\mathrm{UV}} / d z=-0.19-$ $(-0.11)=0.08$ more evolution at the bright end than the faint end (using the scalings from Table 4: see also Yoshida et al. 2006; Bouwens et al. 2007, 2008 where such differential trends were first discussed). This would suggest an increase of $8_{-4}^{+15} \times$ in the luminosity density from $z \sim 10$ to $z \sim 6$ to -17 mag. Integrating the $z \sim 6 \mathrm{~B} 15 \mathrm{LF}$ to $-17 \mathrm{mag}$ and accounting for this evolution, we estimate a UV luminosity

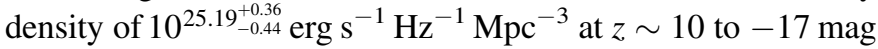
(Figure 8). This estimate should be regarded as an upper limit, as any expected mild evolution in $f_{\text {esc }}$ or $\xi_{\text {ion }}$ (Siana et al. 2010; Hayes et al. 2011; Bouwens et al. 2014: but see also R13) would likely act to lower the derived luminosity density at $z \sim 10$.

How does this luminosity density compare with a simple extrapolation of the $z=4-8$ LF results to $z \sim 10$ ? Adopting the $d \log _{10} \rho_{\mathrm{UV}} / d z=-0.19 \pm 0.04$ scaling implied by the LF results of B15 (Table 4), the extrapolated LF density at $z \sim 10$ is $10^{25.34} \pm 0.10 \mathrm{erg} \mathrm{s}^{-1} \mathrm{~Hz}^{-1} \mathrm{Mpc}^{-3}$ to $-17 \mathrm{mag}$ (see light-blueshaded contour in Figure 8). The luminosity density we infer is consistent with this extrapolation (similar to recent results by Coe et al. 2013; McLeod et al. 2015, or the Oesch et al. 2014b results over the first Frontier Field). However, it is also consistent at $1 \sigma$ with the $(1+z)^{-10.8}$ evolution found by Oesch et al. (2014) at $z>8$, which suggests a luminosity density $10^{25.1 \pm 0.3} \mathrm{erg} \mathrm{s}^{-1} \mathrm{~Hz}^{-1} \mathrm{Mpc}^{-3}$. This is particularly the case, since $z>6$ galaxies may be more efficient at releasing ionizing radiation into the IGM due to evolution in $f_{\text {esc }}$ or $\xi_{\text {ion }}$ (both of which have been speculated to increase at $z>6$ : Siana et al. 2010; Hayes et al. 2011, HM12, KF12, Duncan \& Conselice 2015). This makes the present estimate of the UV luminosity density at $z \sim 10$ effectively an upper limit.

\subsection{How the Ionizing Emissivities We Infer Compare with Key Observational Constraints}

Finally, it is useful to compare the results of our preferred models for the evolution of the ionizing emissivity with the key observational constraints we considered, as a check on the overall self-consistency of the constraints. 


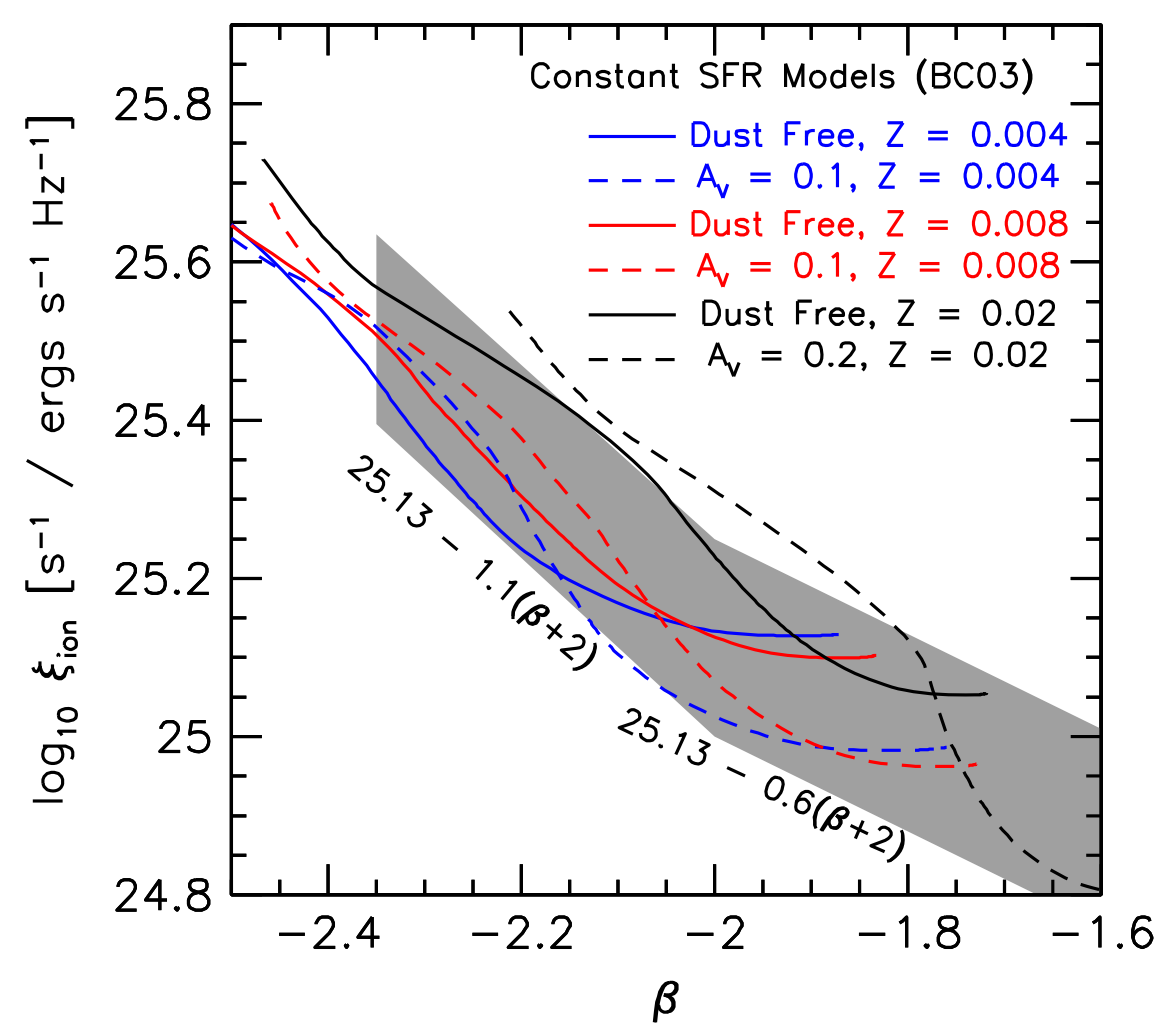

Figure 10. A determination of how the production efficiency $\xi_{\text {ion }}$ of Lyman-continuum photons per unit UV luminosity at $1600 \AA$ A depends on the UV-continuum slope $\beta$. These efficiencies are calculated from the Bruzual \& Charlot (2003) spectral synthesis library assuming a constant star formation rate. We also adopt three different metallicities $\left(0.2 Z_{\odot}, 0.4 Z_{\odot}\right.$, and $\left.Z_{\odot}\right)$ and a wide range in ages $(0.1 \mathrm{Myr}-10 \mathrm{Gyr})$. Both the case of no dust content and $A_{V}=0.1 / 0.2($ Charlot \& Fall 2000$)$ is considered, as indicated on this figure. $\beta$ is computed over the spectral range 1700-2200 $\AA$. The shaded envelopes indicate the approximate dependence of $\xi_{\text {ion }}$ on $\beta$. For completeness the full range of dusty and dust-free tracks are shown, even up to solar metallicity. We recognize that solar metallicities may be unlikely at $z>6$ where results (e.g., Dunlop et al. 2013; Bouwens et al. 2014) suggest that the dust content is very low.

The results for our models preferred at $68 \%$ and $95 \%$ confidence and where reionization finishes at $z=5.9-6.5$ (i.e., when $Q_{\mathrm{H} \text { II }}$ reaches 1 using Equation (2)) are presented in Figure 9. Both the preferred optical depths and reionization histories are shown in this figure. Our ionizing emissivity models are in excellent agreement with all available constraints, including those not considered in deriving the ionizing emissivity (Table 1). A similar version of this figure is presented in Appendix C, but also showing the constraints based on the WMAP nine-year $\tau$ 's.

As in other simple models of cosmic reionization (R13, R15), i.e., where inhomogeneities in the IGM are not treated, we are not able to reproduce observations which suggest incomplete reionization to $z=5-6$, i.e., $Q_{\mathrm{H} \mathrm{I}} \approx$ $1-10^{-4}$. Correctly reproducing the end of reionization would require careful simulations over cosmologically significant volumes, with voids and overdensities, and require the treatment of radiative transfer effects.

Interestingly enough, the optical depths allowed at $68 \%$ confidence only include the lower $50 \%$ of the values allowed by Planck and do not significantly extend above $\tau$ 's of 0.066 . This appears to be the direct result of the constraint we apply on the filling factor of ionized hydrogen at $z=8$ from S14.

If we do not consider the constraint from S14 and instead suppose that $Q_{\mathrm{H} \text { II }}=0.55 \pm 0.20$ at $z \sim 8$ (resulting in an evolution of the ionizing emissivity illustrated by the shadedgreen region in the lower-left panel of Figure 2), the Thomson optical depths we derive span the range $0.060-0.082$. This demonstrates the value of continued work on quantifying the prevalence of Ly $\alpha$ emission in $z \sim 8$ galaxies (e.g., S14) and also constraining the ionizing state of the universe at $z \sim 8$ using other methods (e.g., as Bolton et al. 2011 do at $z \sim 7.1$ using the damping wing of Ly $\alpha$ observed against the spectrum of a bright quasar).

\section{DISCUSSION AND SUMMARY}

Here we have combined the new measurements of the Thompson optical depth $\tau$ from Planck (PC15) with a new approach that focuses on inferring the cosmic ionizing emissivity $\dot{N}_{\text {ion }}$ and its evolution over the reionization epoch from a variety of observables (building of course on significant earlier work by e.g., Madau et al. 1999; Bolton \& Haehnelt 2007; KF12; R13; Becker \& Bolton 2013; R15: see also Mitra et al. 2011, 2012). This approach has allowed us to first gain insight into the allowed evolution of the sources driving reionization, without immediately making assumptions about their nature. We then assess the implications of the derived evolution for the ionizing emissivity and compare with the evolution seen in the luminosity density $\rho_{\mathrm{UV}}$ for galaxies.

We have derived empirical constraints on the evolution of the ionizing emissivity based on the recently measured Thomson optical depth $\tau$ (PC15) and the $Q_{\mathrm{H} \text { н }}(z)$ 's inferred from quasar absorption spectra and the prevalence of Ly $\alpha$ emission in $z=7-8$ galaxies (Fan et al. 2006b; McGreer et al. 2015; S14). We tabulate in Table 1 the extensive results on the filling factor $Q_{\mathrm{H} \text { II }}$ of ionized hydrogen from the literature 

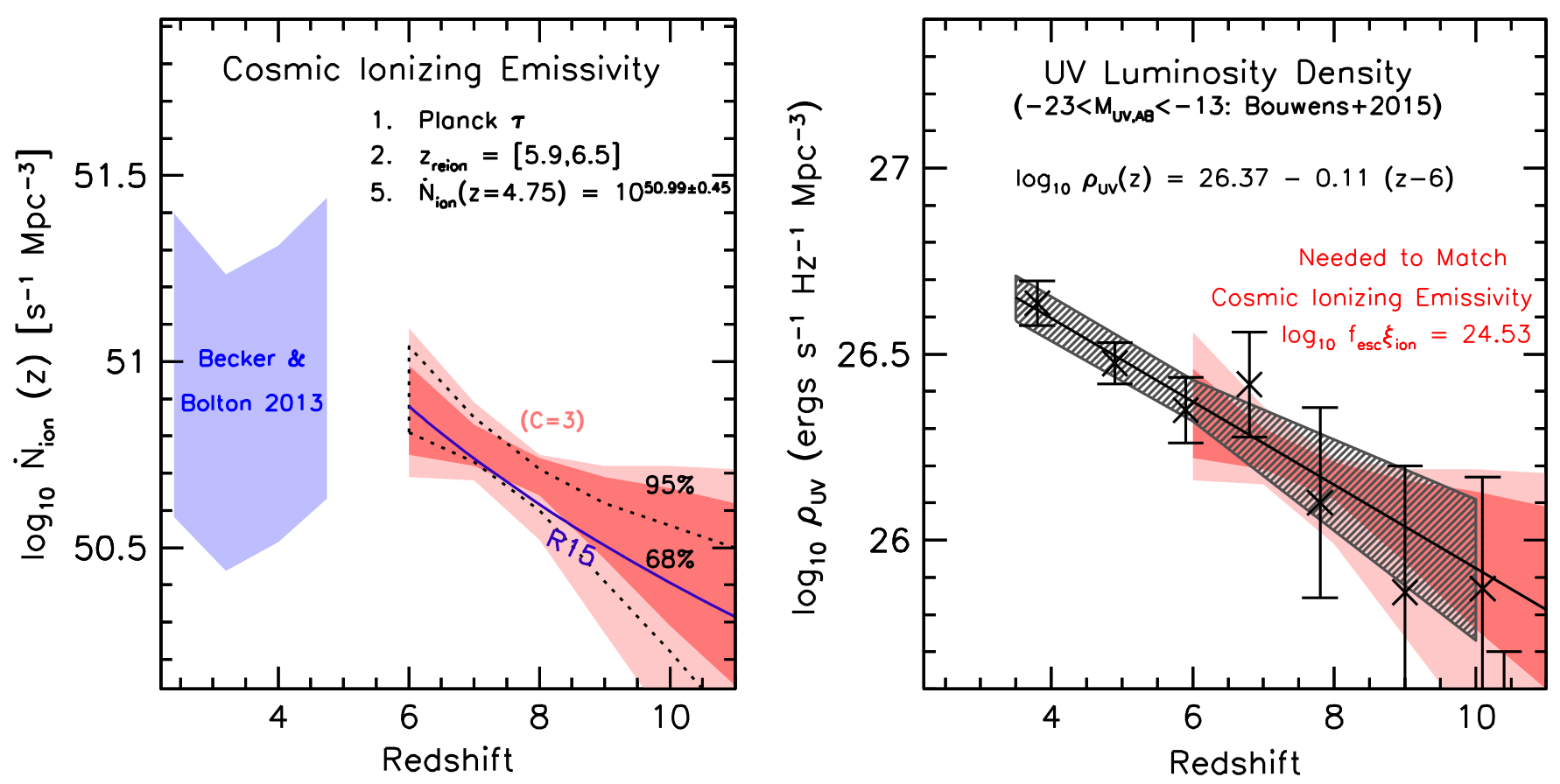

Figure 11. (Left) $68 \%$ and $95 \%$ confidence intervals (red-shaded region) on the evolution of the cosmic ionizing emissivity (assuming $C_{\mathrm{H}}$ II $=3$ ) supposing that reionization is complete at $z=5.9-6.5$, utilizing the latest Planck optical depth measurements (PL15), and enforcing continuity with inferred ionizing emissivity at $z=4.75$ (constraints 1, 2, and 5 from Table 1) and excluding constraints based on the prevalence of Ly $\alpha$ emission in galaxies (Appendix B). The dotted black lines demarcate the $68 \%$ confidence regions for our fiducial determination. See the caption to Figure 6 for a description of other symbols and regions. (Right) comparison of the evolution of the inferred ionizing emissivity with the evolution of the UV luminosity density (black-hatched region) adopting some redshift-independent conversion factor $\log _{10} f_{\mathrm{esc}} \xi_{\text {ion }}=24.53$. As before, the evolution of the ionizing emissivity is very similar to that found for the luminosity density of galaxies in the rest-frame UV. While this again suggests that star-forming galaxies drive the reionization of the universe, this figure demonstrates that this conclusion does not depend on whether or not we make use of constraints that involve $\operatorname{Ly} \alpha$ emission from $z>6$ galaxies.

that allow us to constrain the evolution of the ionizing emissivity $\dot{N}_{\text {ion }}$ (see also Figure 1).

We demonstrate that the evolution in the cosmic ionizing emissivity at $z>6$, i.e., $\left(d \log _{10} \dot{N}_{\text {ion }} / d z\right)_{z=8}=-0.15_{-0.11}^{+0.08}$ $\left(\left(d \log _{10} \dot{N}_{\text {ion }} / d z\right)_{z=8}=-0.19_{-0.11}^{+0.09}\right.$ for a flat prior in $\left(d \log _{10} \dot{N}_{\text {ion }} / d z\right)_{z=8}$ and $\left.\log _{10} \dot{N}_{\text {ion }}(z=8)\right)$, is matched by similar evolution in the UV luminosity density (after extrapolation to $-13 \mathrm{mag}$ ), $\quad$ i.e., $\quad d \log _{10} \rho_{\mathrm{UV}} / d z=$ $-0.11 \pm 0.04$. By contrast, use of the nine-year WMAP optical depths $\tau=0.089 \pm 0.014$ (Bennett et al. 2013) to derive the evolution in the emissivity yields $\left(d \log _{10} \dot{N}_{\text {ion }} / d z\right)_{z=8}=$ $0.00_{-0.06}^{+0.03}$ (Table 3).

This is the first time this similar evolution has been shown quantitatively in this manner and builds on the well-known case that galaxies taken together can provide the UV ionizing radiation needed for reionization (but see also R15). This result further supports the view that star formation in early galaxies drives the reionization of the universe (Section 4.2).

This conclusion is further strengthened by the fact that the requisite conversion factor from the UV luminosity density to the ionizing emissivity $\left(\xi_{\text {ion }} f_{\text {esc }}=10^{24.50} \mathrm{~s}^{-1} /\left(\mathrm{erg} \mathrm{s}^{-1} \mathrm{~Hz}^{-1}\right)\right)$ is consistent with physically plausible values for the escape fraction $f_{\text {esc }}$ and $\xi_{\text {ion }}$, for a faint-end limit $M_{\text {lim }}$ to the UV LF of -13 mag and a clumping factor $C_{\mathrm{H} \text { II }}=3$. We calculate that this conversion factor has an approximate uncertainty of $\sim 0.1$ dex (Section 4.2) based on the uncertainties in the normalization of the cosmic ionizing emissivity and the UV luminosity density. We also present a generalization of this constraint for other values of $M_{\mathrm{lim}}$ and $C_{\mathrm{H}}$ II , i.e., Equation (5). We manipulate this constraint to provide a general formula for deriving $f_{\text {esc }}$ for a wide range of different values for $\xi_{\text {ion }}, M_{\text {lim }}$, $C_{\mathrm{H} \text { II }}$ assuming that galaxies drive the reionization of the universe (see Equation (6) and Table 5).

This is also one of the first analyses where the uncertainty on this conversion factor has been estimated from constraints on the ionization state of the universe at $z>5.9$ and is the direct result of the inferences we make regarding the evolution of the ionizing emissivity (but see also Mitra et al. 2013). [KF12 achieved a similar constraint on the conversion factor at $z \sim 4$ from the ionizing emissivity estimates based on observations of the Ly $\alpha$ forest.] Despite the size of these uncertainties, the consistency with physically plausible values of $f_{\text {esc }}, \xi_{\text {ion }}$, and $M_{\mathrm{lim}}$ was not assured a priori and provides confidence that galaxies play a dominant role in reionization.

We also consider quasars/AGNs as potential sources of the ionizing radiation. However, as for most previous assessments, quasars/AGNs appear unlikely to be the dominant source of the ionizing UV radiation, even under rather generous assumptions about the contributions of faint AGNs. There is simply little evidence they show the required emissivities nor redshift dependence to match that found for the ionizing emissivity (Section 4.3: but see Giallongo et al. 2015).

Assuming no change in the production efficiency of UV continuum photons or ionizing photons, our constraints on the evolution of the ionizing emissivity can be used to estimate the UV luminosity density at $z \sim 10$. We show in Section 4.4 that it is $8_{-4}^{+15} \times$ lower than at $z \sim 6$. As we note, there is also the possibility, due to either evolution in $\xi_{\text {ion }}$ or $f_{\text {esc }}$, that $z>6$ galaxies are more efficient (per unit UV luminosity) at releasing Lyman-continuum radiation into the IGM than $z \sim 6$ galaxies. If that is the case, then this estimate on the UV luminosity 

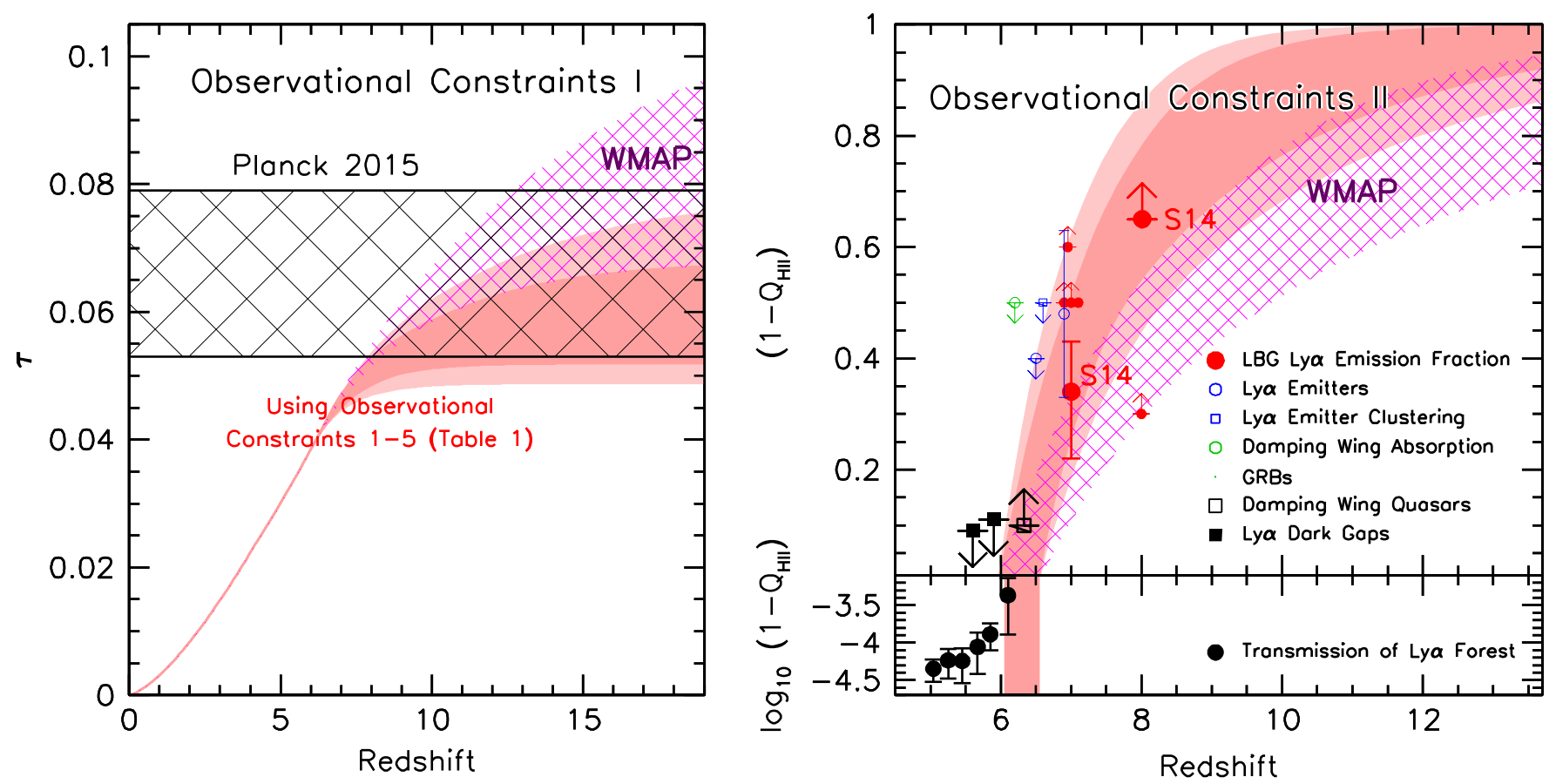

Figure 12. (Left) Comparison of the constraints on the Thomson optical depth $\tau$ provided by the Planck three-year results (PC15: cross-hatched black region) and the cumulative optical depths preferred at $68 \%$ and $95 \%$ confidence by our models of the ionizing emissivity evolution including new constraints from Planck. The magenta-hatched region show the constraints that would be provided by the WMAP results (68\% confidence). (Right) Shown are constraints on the filling factor of ionized hydrogen $Q_{\mathrm{H} \text { п }}$ as a function of redshift and the evolution preferred at $68 \%$ and $95 \%$ confidence by our models of the ionizing emissivity evolution. The magenta-hatched region indicates the range of $Q_{\mathrm{H} \text { п }}$ allowed at 68\% confidence for the WMAP nine-year $\tau$ measurement $(0.089 \pm 0.014)$. Similar to Figure 9 .

density of $z \sim 10$ galaxies is an upper limit. The uncertainty on this estimate, however, is large enough that it cannot help to resolve the question about the potential change in the slope of the luminosity density at around $z \sim 8$ as first identified by Oesch et al. (2012).

The recent remarkable observations from Planck (PC15) provide a fresh opportunity to re-evaluate the role of galaxies in cosmic reionization (see also R15; Mitra et al. 2015). We have taken advantage of these new Planck results on the Thompson optical depth, as well as a decade of observations of galaxies and quasars that provide constraints on the filling factor of ionized hydrogen as a function of redshift (see Section 4.5), to take a further step. We show not only that reionization is consistent with being driven by the UV radiation from galaxies, as many others have demonstrated, but also that the evolution of the ionizing emissivity (from $z \sim 4$ to $z \sim 10$ ) matches a similar trend in the UV luminosity density (see Figure 6).

The results here substantially strengthen the growing consensus that early galaxies are the key to reionization. These results on cosmic reionization from current state-of-the-art microwave background probes (PC15) combined with deep probes of faint $z>6$ galaxies provided by the Hubble Space Telescope (HST) (e.g., Bouwens et al. 2011; McLure et al. 2013; R13; B15; Atek et al. 2015) have demonstrated the power of combining data from a wide range of major missions, and highlight the upcoming opportunities with James Webb Space Telescope for probing deeply into the reionization epoch.

We are grateful to George Becker, Andrea Ferrara, Kristian Finlator, Marijn Franx, Andrei Mesinger, Sourav Mitra, Joop
Schaye, and Michele Trenti for conversations related to this work. This paper owes its existence to Andrei Mesinger's tasking the first author with writing a chapter for a future book. We acknowledge the support of NASA grant NAG5-7697, NASA/STScI grant HST-GO-11563, and a NWO vrijcompetitie grant 600.065.140.11N211.

\section{APPENDIX A \\ PRODUCTION EFFICIENCY FOR LYMAN CONTINUUM PHOTONS PER UNIT UV LUMINOSITY}

In this section, we determine how the production efficiency per unit UV luminosity $\xi_{\text {ion }}$ depends on the UV-continuum slope $\beta$ (where $\beta$ is defined such that $f_{\lambda} \propto \lambda^{\beta}$ ). Given that observations only allow for a constraint on the product of the two unknowns $\xi_{\text {ion }} f_{\text {esc }}$ (and perhaps more generally the production of the four unknowns $\left.\xi_{\text {ion }} f_{\text {esc }} f\left(\mathrm{M}_{\text {lim }}\right) \mathrm{C}_{\mathrm{H} \mathrm{II}} / 3\right)^{-0.3}$; see Equation 6 from Section 4.2) the choice of a physically plausible value for $\xi_{\text {ion }}$ ensures that the $f_{\text {esc }}$ we infer is also of more relevance.

Following a similar procedure to that executed in R13, we can estimate the efficiency parameter $\xi_{\text {ion }}$ by considering a variety of different ages, metallicities, and dust content for starforming galaxies at $z>6$. For convenience, the star-formation rate of our model galaxies is assumed to be constant. The Charlot \& Fall (2000) dust model is assumed, and we also leverage the spectral synthesis models of Bruzual \& Charlot (2003). The UV-continuum slope $\beta$ is derived from the model spectra over the wavelength 1700 and $2200 \AA$, consistent with the position of the broadbands used to derive $\beta$ for $z \sim 7$ galaxies (e.g., Bouwens et al. 2010, 2014; Dunlop et al. 2013). 
The conversion factors and $\beta$ 's computed for many different model spectra are presented in Figure 10.

Fitting the envelope of derived conversion factors $\xi_{\text {ion }}$ versus $\beta$, we find $10^{25.13-1.1(\beta+2)} \mathrm{s}^{-1} /\left(\mathrm{erg} \mathrm{s}^{-1} \mathrm{~Hz}^{-1}\right)$ for $\beta<-2$ and $10^{25.13-0.6(\beta+2)} \mathrm{s}^{-1} /\left(\mathrm{erg} \mathrm{s}^{-1} \mathrm{~Hz}^{-1}\right)$ for $\beta>-2$, with an approximate width of this distribution of \pm 0.125 in $\log _{10} \xi_{\text {ion }}$.

\section{APPENDIX B EVOLUTION INFERRED FOR COSMIC IONIZING EMISSIVITY EXCLUDING CONSTRAINTS BASED ON THE PREVALENCE OF LY $\alpha$ EMISSION IN $z>6$ GALAXIES}

As discussed extensively in the text and elsewhere (e.g., Choudhury et al. 2015; Mesinger et al. 2015), large uncertainties exist in the use of $\operatorname{Ly} \alpha$ emission from galaxies to constrain the ionization state of the universe at $z>6$. Not only do the observational inferences depend on the intrinsic velocity offset of Ly $\alpha$ emission (e.g., Mesinger et al. 2015; Stark et al. 2015), but it is dependent on details of the radiative transfer, inhomogeneities in the IGM, the ionizing background, all of which rely on high-quality simulations of the reionization process (and where it can be challenging to include all of the relevant physics).

As such, it is perhaps useful to derive the evolution in the cosmic ionizing emissivity without relying on observational constraints that involve the prevalence of Ly $\alpha$ emission in $z \sim 7$ and $z \sim 8$ galaxies. We therefore repeat the exercise we performed in Section 4.1 but excluding constraints 3-4 from Table 1.

Our derived constraints on the evolution of the ionizing emissivity (68\% and $95 \%$ confidence intervals) are shown in the left panel of Figure 11. The equivalent $\left(d \log _{10} \dot{N}_{\text {ion }} / d z\right)_{z=8}$, after marginalizing over parameter space and weighting by $|\nabla \tau|$ (a flat prior in $\tau$ : see Section 4.1), is $-0.09_{-0.13}^{+0.06}$ (Table 3), which is $0.5 \sigma$ shallower than in our fiducial analysis (dotted black line gives the $68 \%$ confidence intervals) where we fold in the constraints from the observed Ly $\alpha$ fraction. The right panel in Figure 11 compares these emissivity results with the evolution of the UV luminosity density (see also Figure 6).

Here again the evolution we infer for the ionizing emissivity is in excellent agreement with the evolution found in the UV luminosity density (B15). As in our fiducial analysis, the similarity of the evolution found for these two quantities suggests that star-forming galaxies provide the ionizing photons which drive the reionization of the universe. The exercise we present in this appendix shows that this conclusion does not depend on our use of constraints that involve the prevalence of Ly $\alpha$ emission from $z>6$ galaxies.

\section{APPENDIX C \\ COMPARISON BETWEEN THE INTEGRATED $\tau$ 'S AND REIONIZATION HISTORIES IMPLIED BY PLANCK AND THE WMAP NINE-YEAR RESULTS}

For context and to illustrate the gains from Planck, we also Figure 12 showing the filling factor evolution of ionized hydrogen, $Q_{\mathrm{H} \text { II }}$, and integrated Thomson optical depths $\tau$ 's as inferred from the WMAP nine-year $\tau$ measurement and assuming reionization is complete at $z=5.9-6.5$ (magenta hatched region: $68 \%$ confidence).

\section{REFERENCES}

Alavi, A., Siana, B., Richard, J., et al. 2014, ApJ, 780, 143

Alvarez, M. A., Finlator, K., \& Trenti, M. 2012, ApJL, 759, L38

Atek, H., Richard, J., Kneib, J.-P., et al. 2015, ApJ, 800, 18

Barone-Nugent, R. L., Wyithe, J. S. B., Trenti, M., et al. 2015, MNRAS, 450, 1224

Becker, G. D., \& Bolton, J. S. 2013, MNRAS, 436, 1023

Becker, G. D., Bolton, J. S., Haehnelt, M. G., \& Sargent, W. L. W. 2011, MNRAS, 410, 1096

Becker, R. H., Fan, X., White, R. L., et al. 2001, AJ, 122, 2850

Beckwith, S. V. W., Stiavelli, M., Koekemoer, A. M., et al. 2006, AJ, 132,1729

Bennett, C. L., Larson, D., Weiland, J. L., et al. 2013, ApJS, 208, 20

Bolton, J. S., \& Haehnelt, M. G. 2007, MNRAS, 382, 325

Bolton, J. S., \& Haehnelt, M. G. 2013, MNRAS, 429, 1695

Bolton, J. S., Haehnelt, M. G., Warren, S. J., et al. 2011, MNRAS, 416, L70

Bouwens, R. J., Illingworth, G. D., Blakeslee, J. P., \& Franx, M. 2006, ApJ, 653,53

Bouwens, R. J., Illingworth, G. D., Franx, M., \& Ford, H. 2007, ApJ, 670, 928 Bouwens, R. J., Illingworth, G. D., Franx, M., \& Ford, H. 2008, ApJ, 686, 230 Bouwens, R. J., Illingworth, G. D., Oesch, P. A., et al. 2010, ApJL, 709, L133 Bouwens, R. J., Illingworth, G. D., Oesch, P. A., et al. 2011, ApJ, 737, 90

Bouwens, R. J., Illingworth, G. D., Oesch, P. A., et al. 2012a, ApJL, 752, L5 Bouwens, R. J., Illingworth, G. D., Oesch, P. A., et al. 2012b, ApJ, 754, 83 Bouwens, R. J., Illingworth, G. D., Oesch, P. A., et al. 2014, ApJ, 793, 115 Bouwens, R. J., Illingworth, G. D., Oesch, P. A., et al. 2015, ApJ, 803, 34 Bowler, R. A. A., Dunlop, J. S., McLure, R. J., et al. 2015, MNRAS, 452, 1817 Bruzual, G., \& Charlot, S. 2003, MNRAS, 344, 1000

Bunker, A. J., Wilkins, S., Ellis, R. S., et al. 2010, MNRAS, 409, 855

Cai, Z.-Y., Lapi, A., Bressan, A., et al. 2014, ApJ, 785, 65

Caruana, J., Bunker, A. J., Wilkins, S. M., et al. 2012, MNRAS, 427, 3055

Caruana, J., Bunker, A. J., Wilkins, S. M., et al. 2014, MNRAS, 443, 2831

Charlot, S., \& Fall, S. M. 2000, ApJ, 539, 718

Chen, X., Cooray, A., Yoshida, N., \& Sugiyama, N. 2003, MNRAS, 346, L31 Choudhury, T. R., \& Ferrara, A. 2006, MNRAS, 371, L55

Choudhury, T. R., Puchwein, E., Haehnelt, M. G., \& Bolton, J. S. 2015, MNRAS, 452, 261

Coe, D., Bradley, L., \& Zitrin, A. 2015, ApJ, 800, 84

Coe, D., Zitrin, A., Carrasco, M., et al. 2013, ApJ, 762, 32

Cooke, J., Ryan-Weber, E. V., Garel, T., \& Díaz, C. G. 2014, MNRAS, 441,837

Dijkstra, M., Haiman, Z., Rees, M. J., \& Weinberg, D. H. 2004, ApJ, 601, 666 Dijkstra, M., Mesinger, A., \& Wyithe, J. S. B. 2011, MNRAS, 414, 2139

Duncan, K., \& Conselice, C. J. 2015, MNRAS, 451, 2030

Dunlop, J. S., Rogers, A. B., McLure, R. J., et al. 2013, MNRAS, 432, 3520 Ellis, R. S., McLure, R. J., Dunlop, J. S., et al. 2013, ApJL, 763, L7

Fan, X., Carilli, C. L., \& Keating, B. 2006a, ARA\&A, 44, 415

Fan, X., Strauss, M. A., Becker, R. H., et al. 2006b, AJ, 132, 117

Fan, X., Strauss, M. A., Schneider, D. P., et al. 2001, AJ, 121, 54 Finkelstein, S. L., Papovich, C., Ryan, R. E., et al. 2012a, ApJ, 758, 93 Finkelstein, S. L., Papovich, C., Salmon, B., et al. 2012b, ApJ, 756, 164 Finlator, K., Oh, S. P., Özel, F., \& Davé, R. 2012, MNRAS, 427, 2464 Finlator, K., Oppenheimer, B. D., \& Davé, R. 2011, MNRAS, 410, 1703 Fontana, A., Vanzella, E., Pentericci, L., et al. 2010, ApJL, 725, L205

Giallongo, E., Grazian, A., Fiore, F., et al. 2015, A\&A, 578, A83

Haardt, F., \& Madau, P. 2012, ApJ, 746, 125

Hansen, S. H., \& Haiman, Z. 2004, ApJ, 600, 26

Hathi, N. P., Cohen, S. H., Ryan, R. E., Jr., et al. 2013, ApJ, 765, 88

Hayes, M., Schaerer, D., Östlin, G., et al. 2011, ApJ, 730, 8

Hui, L., \& Haiman, Z. 2003, ApJ, 596, 9

Ishigaki, M., Kawamata, R., Ouchi, M., et al. 2015, ApJ, 799, 12

Keating, L. C., Haehnelt, M. G., Becker, G. D., \& Bolton, J. S. 2014, MNRAS, 438, 1820

Kuhlen, M., \& Faucher-Giguère, C.-A. 2012, MNRAS, 423, 862

Kurczynski, P., Gawiser, E., Rafelski, M., et al. 2014, ApJL, 793, L5

Loeb, A. 2006, SciAm, 295, 46

Loeb, A., \& Barkana, R. 2001, ARA\&A, 39, 19

Mac Low, M.-M., \& Ferrara, A. 1999, ApJ, 513, 142

Madau, P., \& Dickinson, M. 2014, ARA\&A, 52, 415

Madau, P., Haardt, F., \& Rees, M. J. 1999, ApJ, 514, 648

Madau, P., Rees, M. J., Volonteri, M., Haardt, F., \& Oh, S. P. 2004, ApJ, 604, 484

Malhotra, S., \& Rhoads, J. E. 2004, ApJL, 617, L5

McGreer, I. D., Jiang, L., Fan, X., et al. 2013, ApJ, 768, 105

McGreer, I. D., Mesinger, A., \& D'Odorico, V. 2015, MNRAS, 447, 499 
McLeod, D. J., McLure, R. J., Dunlop, J. S., et al. 2015, MNRAS, 450, 3032 McLure, R. J., Dunlop, J. S., Bowler, R. A. A., et al. 2013, MNRAS, 432, 2696 McQuinn, M., Hernquist, L., Zaldarriaga, M., \& Dutta, S. 2007, MNRAS, 381, 75

McQuinn, M., Lidz, A., Zaldarriaga, M., Hernquist, L., \& Dutta, S. 2008, MNRAS, 388, 1101

Mesinger, A., Aykutalp, A., Vanzella, E., et al. 2015, MNRAS, 446, 566

Mesinger, A., \& Furlanetto, S. R. 2008, MNRAS, 386, 1990

Mitra, S., Choudhury, T. R., \& Ferrara, A. 2011, MNRAS, 413, 1569

Mitra, S., Choudhury, T. R., \& Ferrara, A. 2012, MNRAS, 419, 1480

Mitra, S., Choudhury, T. R., \& Ferrara, A. 2015, MNRAS, submitted (arXiv:1505.05507)

Mitra, S., Ferrara, A., \& Choudhury, T. R. 2013, MNRAS, 428, L1

Mortlock, D. J., Warren, S. J., Venemans, B. P., et al. 2011, Natur, 474, 616

Mostardi, R. E., Shapley, A. E., Nestor, D. B., et al. 2013, ApJ, 779, 65

Muñoz, J. A., \& Loeb, A. 2011, ApJ, 729, 99

Nestor, D. B., Shapley, A. E., Kornei, K. A., Steidel, C. C., \& Siana, B. 2013, ApJ, 765, 47

Oesch, P. A., Bouwens, R. J., Illingworth, G. D., et al. 2010, ApJL, 709, L16

Oesch, P. A., Bouwens, R. J., Illingworth, G. D., et al. 2012, ApJ, 745, 110

Oesch, P. A., Bouwens, R. J., Illingworth, G. D., et al. 2013, ApJ, 773, 75

Oesch, P. A., Bouwens, R. J., Illingworth, G. D., et al. 2014, ApJ, 786, 108

Oesch, P. A., Bouwens, R. J., Illingworth, G. D., et al. 2015, ApJ, 808, 104

Oesch, P. A., Carollo, C. M., Stiavelli, M., et al. 2009, ApJ, 690, 1350

Ono, Y., Ouchi, M., Mobasher, B., et al. 2012, ApJ, 744, 83

O'Shea, B. W., Wise, J. H., Xu, H., \& Norman, M. L. 2015, ApJL, 807, L12

Ota, K., Iye, M., Kashikawa, N., et al. 2008, ApJ, 677, 12

Ouchi, M., Shimasaku, K., Furusawa, H., et al. 2010, ApJ, 723, 869

Pawlik, A. H., Schaye, J., \& Dalla Vecchia, C. 2015, MNRAS, 451, 1586

Pawlik, A. H., Schaye, J., \& van Scherpenzeel, E. 2009, MNRAS, 394, 1812

Pentericci, L., Fontana, A., Vanzella, E., et al. 2011, ApJ, 743, 132

Pentericci, L., Vanzella, E., Fontana, A., et al. 2014, ApJ, 793, 113

Planck Collaboration, Ade, P. A. R., Aghanim, N., et al. 2015, arXiv:1502.01589

Reddy, N. A., \& Steidel, C. C. 2009, ApJ, 692, 778

Rees, M. J., \& Ostriker, J. P. 1977, MNRAS, 179, 541
Ricotti, M., \& Ostriker, J. P. 2004, MNRAS, 352, 547

Ricotti, M., Ostriker, J. P., \& Mack, K. J. 2008, ApJ, 680, 829

Robertson, B. E., Ellis, R. S., Furlanetto, S. R., \& Dunlop, J. S. 2015, ApJL, 802, L19

Robertson, B. E., Furlanetto, S. R., Schneider, E., et al. 2013, ApJ, 768, 71 Santos, M. R. 2004, MNRAS, 349, 1137

Schenker, M. A., Ellis, R. S., Konidaris, N. P., \& Stark, D. P. 2014, ApJ, 795,20

Schenker, M. A., Robertson, B. E., Ellis, R. S., et al. 2013, ApJ, 768, 196

Schenker, M. A., Stark, D. P., Ellis, R. S., et al. 2012, ApJ, 744, 179

Schroeder, J., Mesinger, A., \& Haiman, Z. 2013, MNRAS, 428, 3058

Shull, J. M., Harness, A., Trenti, M., \& Smith, B. D. 2012, ApJ, 747, 100

Siana, B., Shapley, A. E., Kulas, K. R., et al. 2015, ApJ, 804, 17

Siana, B., Teplitz, H. I., Ferguson, H. C., et al. 2010, ApJ, 723, 241

Sobacchi, E., \& Mesinger, A. 2015, arXiv:1505.02787

Somerville, R. S., Bullock, J. S., \& Livio, M. 2003, ApJ, 593, 616

Spergel, D. N., Verde, L., Peiris, H. V., et al. 2003, ApJS, 148, 175

Stark, D. P., Ellis, R. S., Chiu, K., Ouchi, M., \& Bunker, A. 2010, MNRAS, 408, 1628

Stark, D. P., Ellis, R. S., \& Ouchi, M. 2011, ApJL, 728, L2

Stark, D. P., Richard, J., Charlot, S., et al. 2015, MNRAS, 450, 1846

Tilvi, V., Papovich, C., Finkelstein, S. L., et al. 2014, ApJ, 794, 5

Topping, M. W., \& Shull, J. M. 2015, ApJ, 800, 97

Totani, T., Kawai, N., Kosugi, G., et al. 2006, PASJ, 58, 485

Trenti, M., Stiavelli, M., Bouwens, R. J., et al. 2010, ApJL, 714, L202

Treu, T., Schmidt, K. B., Trenti, M., Bradley, L. D., \& Stiavelli, M. 2013, ApJL, 775, L29

Vanzella, E., Guo, Y., Giavalisco, M., et al. 2012, ApJ, 751, 70

Weigel, A. K., Schawinski, K., Treister, E., et al. 2015, MNRAS, 448, 3167

Wilkins, S. M., Bunker, A., Coulton, W., et al. 2013, MNRAS, 430, 2885

Wilkins, S. M., Bunker, A. J., Stanway, E., Lorenzoni, S., \& Caruana, J. 2011, MNRAS, 417, 717

Willott, C. J., Delorme, P., Reylé, C., et al. 2009, AJ, 137, 3541

Willott, C. J., Delorme, P., Reylé, C., et al. 2010, AJ, 139, 906

Yan, H., \& Windhorst, R. A. 2004, ApJL, 600, L1

Yoshida, M., Shimasaku, K., Kashikawa, N., et al. 2006, ApJ, 653, 988 